\title{
Delineation of the olive pollen proteome and its allergenome unmasks cyclophilin as a relevant cross-reactive allergen
}

Pablo San Segundo-Acosta ${ }^{\ddagger, \#, ~ C a r m e n ~ O e o-S a n t o s ~}{ }^{\ddagger, \#}$, Sara Benedé ${ }^{\ddagger}$, Vivian de los Ríos ${ }^{\S}$, Ana Navas", Berta Ruiz-Leon", Carmen Moreno", Carlos Pastor-Vargas $\varsigma$, Aurora

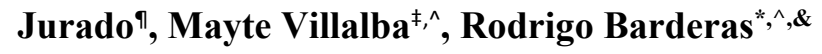

\#, Both authors contributed equally. ^, Co-senior authors

‡Departamento de Bioquímica y Biología Molecular, Facultad de Ciencias Químicas, Universidad Complutense de Madrid, E-28040 Madrid, Spain

${ }^{\S}$ Centro de Investigaciones Biológicas, CSIC, E-28040 Madrid, Spain

"Hospital Universitario Reina Sofía de Córdoba, E-14004 Córdoba, Spain

`Hospital Fundación Jiménez Díaz, E-28040 Madrid, Spain

${ }^{\&}$ Chronic Disease Programme (UFIEC), Instituto de Salud Carlos III, Majadahonda E-28220, Madrid, Spain

*To whom correspondence should be addressed

"Corresponding Author: Rodrigo Barderas, Functional Proteomics Unit, Chronic Disease Programme (UFIEC), Instituto de Salud Carlos III. Majadahonda, Madrid. Spain. 28220.

E-mail: r.barderasm@isciii.es 


\begin{abstract}
Olive pollen is a major allergenic source worldwide for its extensive cultivation. We have combined available genomic data with a comprehensive proteomic approach to get the annotated olive tree (Olea europaea L.) pollen proteome and define its complex allergenome. A total of 1,907 proteins were identified by LC-MS/MS using predicted protein sequences from its genome. Most proteins $(60 \%)$ were predicted to possess catalytic activity and be involved in metabolic processes. In total, 203 proteins belonging to 47 allergen families were found in olive pollen. A peptidyl-prolyl cis-trans isomerase -cyclophilin- produced in Escherichia coli, was found as a new olive pollen allergen (Ole e 15). Most Ole e 15-sensitized patients were children (63\%) and showed strong $\operatorname{IgE}$ recognition to the allergen. Ole e 15 shared high sequence identity with other plant, animal and fungal cyclophilins and presented high IgE cross-reactivity with pollen, plant food and animal extracts.
\end{abstract}

KEYWORDS: Olive pollen proteome, allergenome, in-depth proteomics, allergen, cyclophilin, cross-reactivity 


\section{INTRODUCTION}

The pollen grain has aroused a lot of interest not only for its role as male gametophyte in reproduction (1), but also for its major role as allergen source. Proteomic studies have shown that proteins acting in pollen formation and germination are mainly involved in energy production, defense and protein synthesis and processing (2-4). Moreover, as remarked above, some pollen proteins are capable of triggering airway inflammation and antigenic responses, which in some individuals derive in allergy (5-8). For this reason, proteomic analysis of pollen can be used for allergen identification, as it has been done for several allergenic sources (9-13). However, there is limited genomic information of tree species with allergenic pollen, reducing the potential number of these studies.

Recently, the complete genome of the wild olive (Olea europaea subsp. europaea var. sylvestris -also denominated as oleaster-), a closely related variant of cultivated olive ( $O$. europaea subsp. europaea var. europaea) has been reported (14). Cultivated olive belongs to the Oleaceae family (order Lamiales) and has been traditionally cultivated in the Mediterranean Basin, being the main crop in some areas of Spain, Italy and Greece (15). In addition, olive trees are extensively cultivated worldwide for olive oil and olive fruit production. Several regions of these countries reach extremely high olive pollen counts in the pollination season, becoming olive pollen a major allergenic health problem (16). Until now, thirteen olive pollen allergens, Ole e 1 to Ole e 12 and Ole e 14, have been identified (17-19). However, some allergens remain unidentified due to technical restrictions and limited genomic and proteomic data. Moreover, very little is known about other proteins present in this pollen, which although not allergenic, may well influence the immune response of patients by promoting inflammation (20-22).

In this work, we have performed a comprehensive proteomic analysis of cultivated olive pollen, taking advantage of the wild olive genomic data. By an in-depth bottom-up proteomic approach, olive pollen proteome has been in-depth identified using a Q-exactive mass spectrometer, and functionally catalogued according to Gene Ontology (GO) terms. We next extended our analysis to the definition of the olive pollen allergenome, identifying its allergen 
families by searching the proteomic data with the Allergome, World-Health Organization and International Union of Immunological Societies (WHO/IUIS) and AllFam (the database of allergen families) databases (23-25). Among them, we finally focused the study on the verification of the allergenicity of four proteins non-previously studied in olive pollen because of their potential involvement in cross-reactivity with other allergenic sources. We found cyclophilin, a peptidyl-prolyl cis-trans isomerase as a new relevant olive pollen allergen. Enzyme-linked immunosorbent assay (ELISA), western blot (WB), and Immunoglobulin E (IgE) inhibition assays revealed its significance, high potency and its wide range cross-reactivity with pollen and plant-derived food, dust mites and its human counterparts. 
METHODS

\section{Protein extracts}

Commercial olive pollen from Allergon (Batch Nº32509801, Ängelholm, Sweden) collected in California (US) from a controlled set of cultivated olive trees belonging to different cultivars was used for protein extract preparation for proteomics. Three parallel protein extractions were performed and used later as independent biological replicates. Briefly, for each biological replicate, $300 \mathrm{mg}$ of olive pollen were separately resuspended in $6 \mathrm{~mL}$ of extraction buffer (100 $\mathrm{mM}$ Tris- $\mathrm{HCl} \mathrm{pH}$ 7.3, $150 \mathrm{mM} \mathrm{NaCl}, 2$ mM EDTA, 10\% Glycerol, 1\% Triton X-100, and 1 mM Phenylmethanesulfonyl fluoride (PMSF) with 1x Complete Protease Inhibitor Cocktail (Roche, Basel, Switzerland ). After homogenization, the resultant emulsion was subjected to mild stirring for $90 \mathrm{~min}$ at room temperature. Then, it was transferred to $2 \mathrm{~mL}$ tubes and centrifuged at $12,000 \mathrm{xg}$ for $20 \mathrm{~min}$ at $4^{\circ} \mathrm{C}$. The insoluble material was resuspended again in 1.5 $\mathrm{mL}$ of extraction buffer and stirred for $1 \mathrm{~h}$. After centrifugation, both supernatants were pooled together and a sample analyzed by SDS-PAGE.

Pollen protein extracts and plant-derived food protein extracts were prepared for the other assays according to established protocols (26), and their concentration measured using the Lowry protein assay.

\section{SDS-PAGE, trypsin digestion and LC-MS/MS analysis}

Firstly, $40 \mu \mathrm{g}$ of each extract replicate were separated by 15\% SDS-PAGE and stained with Coomassie Brilliant Blue G 250 (Merck, Darmstadt, Germany). Then, whole lanes were cut into four (replicate 1) or six slices (replicates 2 and 3) prior to in-gel trypsin digestion $(27,28)$. Then, excised bands were cut in small pieces and separately destained with $50 \mathrm{mM}$ ammonium bicarbonate $(\mathrm{ABC}) / 50 \%$ acetonitrile $(\mathrm{ACN})$, dehydrated with $\mathrm{ACN}$ and dried. Samples were then reduced with $10 \mathrm{mM}$ dithiothreitol in $25 \mathrm{mM} \mathrm{ABC}$ and alkylated with iodoacetamide to a final concentration of $50 \mathrm{mM}$. Then, gel pieces were dried, rehydrated with $12.5 \mathrm{ng} / \mathrm{mL}$ porcine trypsin (Thermo Fisher Scientific, Waltham, MA, US) in $50 \mathrm{mM} \mathrm{ABC}$ and incubated overnight 
at $37{ }^{\circ} \mathrm{C}$. Peptides were extracted using $100 \% \mathrm{ACN}$ and $0.5 \%$ trifluoroacetic acid, purified using a Zip Tip with $0.6 \mu \mathrm{L}$ C18 resin (Millipore, Sigma-Aldrich Química SL, Madrid, Spain), and dried. Finally, samples were reconstituted in $5 \mu \mathrm{L} 0.1 \%$ formic acid $/ 2 \%$ ACN before the analysis by nLC-MS/MS in the Proteomics and Genomics Facility of the Centro de Investigaciones Biológicas (CIB-CSIC, Madrid, Spain), a member of ProteoRed-ISCIII network.

All peptide separations were carried out on an Easy-nLC 1000 nano system (Thermo Fisher Scientific). For each analysis, the sample was loaded into a precolumn Acclaim PepMap 100 (Thermo Fisher Scientific) and eluted in a RSLC PepMap C18, $15 \mathrm{~cm}$ long, $50 \mu \mathrm{m}$ inner diameter and $2 \mu \mathrm{m}$ particle size (Thermo Fisher Scientific). The mobile phase flow rate was 300 $\mathrm{nL} / \mathrm{min}$ using a gradient of $0.1 \%$ formic acid in water (solvent $\mathrm{A}$ ) and $0.1 \%$ formic acid and $100 \% \mathrm{ACN}$ (solvent B). The gradient profile was set as follows: 5\%-35\% solvent B for $45 \mathrm{~min}$, $35 \%-100 \%$ solvent B for $5 \mathrm{~min}, 100 \%$ solvent B for $10 \mathrm{~min}$. Four microliters of each sample were injected.

MS analysis was performed using a Q-Exactive mass spectrometer (Thermo Fisher Scientific). For ionization, $2000 \mathrm{~V}$ of liquid junction voltage and $270{ }^{\circ} \mathrm{C}$ capillary temperature was used. The full scan method employed a m/z 400-1500 mass selection, an Orbitrap resolution of 70,000 (at $\mathrm{m} / \mathrm{z} 200$ ), a target automatic gain control (AGC) value of $3 \mathrm{e} 6$, and maximum injection times of $100 \mathrm{~ms}$. After the survey scan, the 10 most intense precursor ions were selected for MS/MS fragmentation. Fragmentation was performed with normalized collision energy of 27 and MS/MS scans acquired with a starting mass of m/z 100. AGC target was $2 \mathrm{e} 5$, resolution of 17,500 (at $\mathrm{m} / \mathrm{z} 200$ ), intensity threshold of $8 \mathrm{e} 3$, isolation window of 2 $\mathrm{m} / \mathrm{z}$ units and maximum IT was $100 \mathrm{~ms}$. Charge state screening was enabled to reject unassigned, singly charged, and greater than or equal to seven protonated ions. A dynamic exclusion time of 20s was used to discriminate against previously selected ions. 


\section{Data processing}

MS data were analyzed with Proteome Discoverer (version 1.4.1.14, Thermo Fisher Scientific) using standardized workflows. Mass spectra raw files were searched against the NCBI Olea europaea var. sylvestris predicted protein database (taxid 158386, version 11/2017, 49,613 RefSeq protein sequence entries) using SEQUEST search engine. Precursor and fragment mass tolerance were set to $10 \mathrm{ppm}$ and $0.02 \mathrm{Da}$, respectively, allowing 2 missed cleavages, carbamidomethylation of cysteines as a fixed modification, methionine oxidation and threonine, tyrosine and serine phosphorylation as a variable modification. Identified peptides were filtered using Percolator algorithm with a q-value threshold of 0.01 (High Confidence Filter settings, FDR $<1 \%$ ) (29). The mass spectrometry proteomics data have been deposited to the ProteomeXchange Consortium via the PRIDE partner repository, with the data set identifiers PXD012334 (Replicate 1), PXD012279 (Replicate 2), and PXD012280 (Replicate 3).

\section{Experimental and statistical design}

The experimental and statistical design for each experiment of the study are described in each subsection and depicted in Figure 1 and Figure S1. Only proteins containing at least two isoform-specific peptides in one of the replicates, or with one high-confidence isoform-specific peptide but detected in the three replicates, were considered in the study. To identify proteins belonging to allergen families, the full sequences of the identified proteins after LC-MS/MS were retrieved using NCBI Batch Entrez. Then, proteins were locally blasted (E-value threshold 1.0E-15 and 1 Blast hit) against all entries of the Allergome database (4,331 entries; UniProt release 10/2018) using the Blast2GO 5 PRO software (5.2.4 version) (30). Sequence similarity was calculated by Blast2GO, and sequence identity was calculated using Clustal Omega (31). Only those proteins meeting the following criteria were considered as potential allergens: matching with an allergen accepted by the WHO/IUIS Allergen Nomenclature Sub-committee (25) with at least $50 \%$ similarity; matching directly with proteins belonging to the same allergen families as proteins meeting the previous criteria, with at least $80 \%$ similarity; matching with proteins already identified as potential allergens in the Oleaceae family. 


\section{Gene Ontology (GO) annotation and prediction of secreted proteins}

To perform the functional GO annotation and KEGG analysis of each protein, we used the Blast2GO 5 PRO software (5.2.4 version). Firstly, we created a database with the curated NCBI RefSeq predicted proteomes from rice (Oriza sativa, taxid:4530), arabidopsis (Arabidopsis thaliana, taxid: 3702), latex (Hevea brasiliensis, taxid: 3981), barrelclover (Medicago truncatula, taxid: 3880), black cottonwood (Populus trichocarpa, taxid: 3694), peach (Prunus persica, taxid: 3760), tomato (Solanum lycopersicum, taxid:4081), potato (Solanum tuberosum, taxid: 4113) and common grape vine (Vitis vinifera, taxid: 3603). GO annotations for these curated species are deposited in Ensembl Plants (32), and accessible through Blast2GO by BioMart (33). Then, olive pollen identified proteins were locally blasted against this database (maximum number of BLAST hits: 1; minimal E-value: 1.0E-50; BLAST descriptor annotator activated, HPS length cut off: 50, rest of parameters: default). GO mapping (GOA version 09.2018, default settings) and annotation (taxonomy filter: taxa: 33090, Viridiplantae) were performed. Additionally, InterProScan (IPS, default settings) analysis was run (34), and the annotated IPS GOs were merged with previous GOs. Kyoto Encyclopedia of Genes and Genomes (KEGG) Enzyme codes were also annotated. Further information about molecular pathways was obtained by KEGG analysis (35). For Fisher's exact test enrichment analysis of molecular function and biological process GOs, the obtained olive pollen annotation was tested against the whole $O$. europaea var. sylvestris predicted proteome, and annotated using the same parameters as described above for pollen proteins. Only GO terms with p-value $<1.0 \mathrm{E}-10$ were considered as enriched.

Secreted proteins were predicted using the SignalP 4.1 (Organism group: eukaryotes; Dcutoff value 0.45 for Signal P-noTM and TM networks) (36).

\section{Analytical procedures}

1D SDS-PAGE (15\% or 17\% gels) were stained with Coomassie Blue R-250 (Sigma-Aldrich, St.Louis, MO, US) or transferred onto nitrocellulose membranes. Molecular mass calculations 
were performed using unstained protein biomarkers SM0431 (Fermentas, Waltham, MA, US) or pre-stained protein molecular weight markers (Precision Plus Protein All Blue and Prestained Protein Standards low range from Bio-Rad, Hercules, CA, US).

For two-dimensional gel electrophoresis (2DE), isoelectric focusing of $80 \mu \mathrm{g}$ of olive pollen extract was achieved under reducing conditions with $3 \mathrm{mM}$ tributylphosphine in a PROTEAN IEF Cell (Bio-Rad) using pH 3-10, 7 cm length linear ReadyStrip IPG gels (Bio-Rad). After isoelectrofocusing, proteins were separated by $17 \%$ SDS-PAGE under reducing conditions in the presence of $50 \mathrm{mM}$ DTT and $3.7 \%$ iodoacetamide and transferred onto nitrocellulose membranes (Amersham Biosciences, Little Chalfont, UK) according to Towbin method for WB.

Protein concentration of the purified proteins was calculated by measuring the absorbance at $280 \mathrm{~nm}$ using a DU-7 spectrometer (Beckman, Barcelona, Spain) after theoretical extinction coefficient calculation with the ProtParam tool from ExPASy. rOle e 15 concentration was calculated using the experimental extinction coefficient $\left(\mathrm{E}^{0.1 \%}\right)$ of 0.616 obtained after amino acid analysis of $5 \mu \mathrm{g}$ and $10 \mu \mathrm{g}$ of purified protein in duplicates with a BioChrom 30 Amino Acid analyzer (Harvard Bioscience, Inc., Holliston, MA, US).

\section{Patients' sera and antibodies}

A total of 146 sera of patients allergic to olive pollen were used to carry out the study. All samples were handled anonymously according to the ethical and legal guidelines of the Complutense University of Madrid (CEI PI 49) and Reina Sofia University Hospital (ref. 3033). Written informed consent was obtained from all patients. Sera from olive pollen allergic patients according to their positive olive pollen skin prick test and positive profile by ImmunoCAP 250 (Thermo Fisher Scientific, Uppsala, Sweden) were obtained from two different Spanish populations (Córdoba and Madrid, Spain) according to standard operating procedures. Values $>0.35 \mathrm{kU} / \mathrm{L}$ by ImmunoCAP were considered positive. Skin prick tests (SPT) were performed according to standard procedures. A wheal diameter $<3 \mathrm{~mm}$ was considered negative. All patients included in this study reported an immediate IgE-mediated 
allergic reaction and a positive SPT with olive pollen. As a summary of the pollen sensitization of each population: sera from Madrid $(\mathrm{n}=42)$ were all co-sensitized to Lolium perenne, whereas sera from Córdoba $(n=104)$ were sensitized almost exclusively to olive pollen (Table S1). All patients presented asthma or rhinoconjunctivitis.

Rabbit polyclonal antiserum against rOle e 15 was obtained by injections of the protein (100 $\mu \mathrm{g}$ ) in complete Freud's adjuvant, accomplishing the ethics guidelines of the Fundación Jiménez Díaz Hospital (Madrid, Spain). Horseradish peroxidase-labeled goat polyclonal antibody against rabbit IgG was purchased from Bio-Rad. Horseradish peroxidase-labeled mouse anti-human IgE was purchased from Southern Biotech (Birmingham, AL, US).

\section{Circular Dichroism spectroscopic analyses, structural 3D modelling and alignments}

The CD spectrum of rOle e $15(0.2 \mu \mathrm{g} / \mu \mathrm{L})$ in $20 \mathrm{mM}$ phosphate buffer, $\mathrm{pH} 7.0$ was recorded in the far-UV (190-260 nm) on a JASCO J-715 spectropolarimeter (Japan Spectroscopic Co., Tokyo, Japan) using a $0.1 \mathrm{~cm}$ optical-path quartz cuvette and at $20^{\circ} \mathrm{C}, 85^{\circ} \mathrm{C}$ and after cooling down to $20^{\circ} \mathrm{C}$. The deconvolution of the obtained spectra was performed using the CDNN software. Thermal unfolding was followed measuring the ellipticity at $226 \mathrm{~nm}$ heating (20 to $85^{\circ} \mathrm{C}$ ) or cooling $\left(85\right.$ to $\left.20^{\circ} \mathrm{C}\right)$ at $0.5^{\circ} \mathrm{C} / \mathrm{min}$ with a computer controlled water-bath. Far UV spectra were baseline subtracted and represented as mean residue molecular ellipticity.

Sequence identity and similarity of the Ole e 15 amino acid sequence against other plant, animal and fungal cyclophilins were calculated using DIALIGN (Bielefeld Bioinformatics Service, Universität Bielefeld, Germany). The results were visualized using the GeneDoc program. Known cyclophilin structures were downloaded from PDB. Structural models in PDB format were generated using the ExPASy Swiss-Model tool with human (PDB: 1OCA) and Cat r 1 (PDB: 2mc9) as templates. Final 3D-structure models were visualized with PyMOL (Schrödinger, LCC, New York, US). The electrostatic surface potential of rOle e 15 was calculated by the Adaptive Poisson Boltzmann Solver (APBS) program (37) and visualized with PyMOL. 


\section{Immunological characterization}

Indirect ELISA was performed in 96-well plates (Costar, Corning, New York, US) coated with $0.1 \mu \mathrm{g}$ recombinant protein per well, according to previously optimized protocols $(18,38)$, using individual serum samples diluted 1:10.

WB analysis of human IgE bound to alternatively $0.5 \mu \mathrm{g}$ rOle e 15 , or $40 \mu \mathrm{g}$ of olive pollen extract, immobilized onto nitrocellulose membranes was performed incubating with individual human sera (1:10) and horseradish peroxidase-labeled mouse anti-human $\operatorname{IgE}$ Fc $(1: 1,000)$. Binding of specific polyclonal antisera against rOle e 15 was detected by goat anti-rabbit $\operatorname{IgG}$ horseradish peroxidase-labeled antibody $(1: 3,000)$. Chemiluminescent signal was developed using ECL-Western blotting reagent (Amersham Bioscience) or WesternBright ${ }^{\mathrm{TM}}$ QUANTUM (Advansta Inc., San Jose, California, US) reagents.

For the $\operatorname{IgE}$ inhibition experiments with indicated protein extracts, a pool of sera $(n=5$ for $\mathrm{WB}$; $\mathrm{n}=6$ for ELISA) was pre-incubated with $5 \mathrm{mg} / \mathrm{mL}$ for $\mathrm{WB}$; or alternatively 0.25 and 2.5 $\mathrm{mg} / \mathrm{mL}$ for ELISA of the inhibiting extracts for $2 \mathrm{~h}$ at room temperature and shaking. The following steps of the ELISA or WB experiments were performed as described above and the results shown as percentage of inhibition $(18,38)$. For the $\operatorname{IgE}$ inhibition experiments using purified proteins, serum was pre-incubated with $10 \mu \mathrm{g}$ of rOle e 15 , nOle e 1 or both. IgE reactivity was visualized as previously described $(18,35)$. Inhibition values were calculated as percentage in comparison to the non-inhibited control using the formula $\left[\left(1-\mathrm{OD}_{492} \mathrm{~nm}\right.\right.$ with inhibitor/ $\mathrm{OD}_{492 \mathrm{~nm}}$ without inhibitor)*100] by ELISA and as signal lost in comparison to a noninhibited control (No inhibition) by WB after densitometry of the bands using the Quantity One 1-D Analysis software (Bio-Rad Laboratories Inc., Madrid, Spain).

\section{Cloning of the putative allergens from $O$. europaea pollen, expression and purification}

Total RNA purification from pollen was performed using TRIzol and RNeasy Plant MiniKit (Qiagen, Hilden, Germany). Total RNA quality and concentration were analyzed using 2100 Bioanalyzer (Agilent Genomics). cDNA amplification was performed using the SMARTer ${ }^{\circledR}$ 
RACE 5'/3' kit (Clontech, TaKara Bio Company, Mountain View, CA,US) or the SuperScript III First-Strand Synthesis System (Thermo Fisher Scientific). Different degenerate and nondegenerate oligonucleotides (Table S2) were synthesized based on the available proteomic and genomic data. PCR products were cloned into the pCR2.1 plasmid using the TOPO TA Cloning kit (Invitrogen, Groningen, The Netherlands) and sequenced. Then, specific overlapping oligonucleotides (Table S2) were designed to subclone the cDNAs encoding the complete proteins into the pET28a plasmid (Novagen, Billerica, MA, USA) as N-terminal His 6 -tagged proteins using, alternatively, the NEBuilder ${ }^{\circledR}$ HiFi DNA Assembly Mix (New England Biolabs, Ipswich, Massachusetts, US) or the InFusion ${ }^{\circledR}$ HD Cloning Kit (Clontech) following the manufacturer instructions.

The pET28a/cDNA constructs were used to transform BL21 (DE3) E. coli cells. $10 \mathrm{~mL} 30$ $\mu \mathrm{g} / \mathrm{mL}$ kanamycin/LB cultures containing the transformed cells were grown overnight and then, diluted ten times and grown until $\mathrm{OD}_{600 \mathrm{~nm}}$ reached 0.7 . Finally, protein production was induced with $0.4 \mathrm{mM}$ isopropyl thio- $\beta$-D-thiogalactoside (IPTG) and grown at $30^{\circ} \mathrm{C} 230 \mathrm{rpm}$ for $16 \mathrm{~h}$. Then, the cultures were centrifuged at $6,000 \mathrm{x} g$ for $20 \mathrm{~min}$ at $4^{\circ} \mathrm{C}$. Proteins were purified by gravity-flow chromatography from the soluble fraction of cell lysates using Ni-NTA agarose (Qiagen) following the manufacturer instructions with minor modifications. Briefly, cell pellets corresponding to $250 \mathrm{~mL}$ E. coli culture were resuspended in $10 \mathrm{~mL}$ lysis buffer $(300 \mathrm{mM}$ $\mathrm{NaCl}, 1 \mathrm{mM}$ PMSF, $50 \mathrm{mM}$ phosphate buffer $\mathrm{pH} 8.0$ ), distributed in $1 \mathrm{~mL}$ aliquots and disrupted by five cycles of subsequent freezing in liquid $\mathrm{N}_{2}$ for $40 \mathrm{~s}$ and thawing at $42^{\circ} \mathrm{C}$ in a water bath for $5 \mathrm{~min}$. Then, soluble fractions obtained by centrifugation at $6,000 \mathrm{xg}$ for $20 \mathrm{~min}$ at $4^{\circ} \mathrm{C}$ were incubated $16 \mathrm{~h}$ at $4^{\circ} \mathrm{C}$ in a $15 \mathrm{~mL}$ conical centrifuge tube with $500 \mu \mathrm{L}$ Ni-NTA (CliniSciences, Nanterre, France) agarose previously pre-incubated three times with $5 \mathrm{~mL}$ lysis buffer. Then, Ni-NTA agarose was washed five times for $10 \mathrm{~min}$ at $4{ }^{\circ} \mathrm{C}$ with $5 \mathrm{~mL}$ wash buffer (10 mM imidazole, $300 \mathrm{mM} \mathrm{NaCl}, 50 \mathrm{mM}$ phosphate buffer $\mathrm{pH} \mathrm{8.0)} \mathrm{recovering} \mathrm{each} \mathrm{time} \mathrm{the}$ resin by centrifugation at $4,000 \times \mathrm{g}$ for $10 \mathrm{~min}$ at $4^{\circ} \mathrm{C}$. Proteins were then eluted with $5 \mathrm{~mL}$ elution buffer ( $500 \mathrm{mM}$ imidazole, $300 \mathrm{mM} \mathrm{NaCl}, 50 \mathrm{mM}$ phosphate buffer $\mathrm{pH} 8.0$ ) for $20 \mathrm{~min}$ at $4^{\circ} \mathrm{C}$ on rotation. To avoid the presence of traces of Ni-NTA agarose in the eluted fractions, a 
final centrifugation step was performed at $12,000 \mathrm{xg}$ for $5 \mathrm{~min}$ at $4^{\circ} \mathrm{C}$. Finally, purified proteins were desalted onto PD10 desalting columns (GE Healthcare, Chicago, IL, US) with $20 \mathrm{mM}$ $\mathrm{ABC}$, quantified, aliquoted, lyophilized and stored at $-20^{\circ} \mathrm{C}$ until use. 


\section{RESULTS}

\section{Definition of the olive pollen proteome by proteomics and bioinformatics}

In the present study, we have used an in-depth bottom-up proteomic approach to analyze the proteomic profile of olive pollen, to classify its allergen families and define its allergenome, and to characterize new olive pollen allergen candidates (Figure 1).

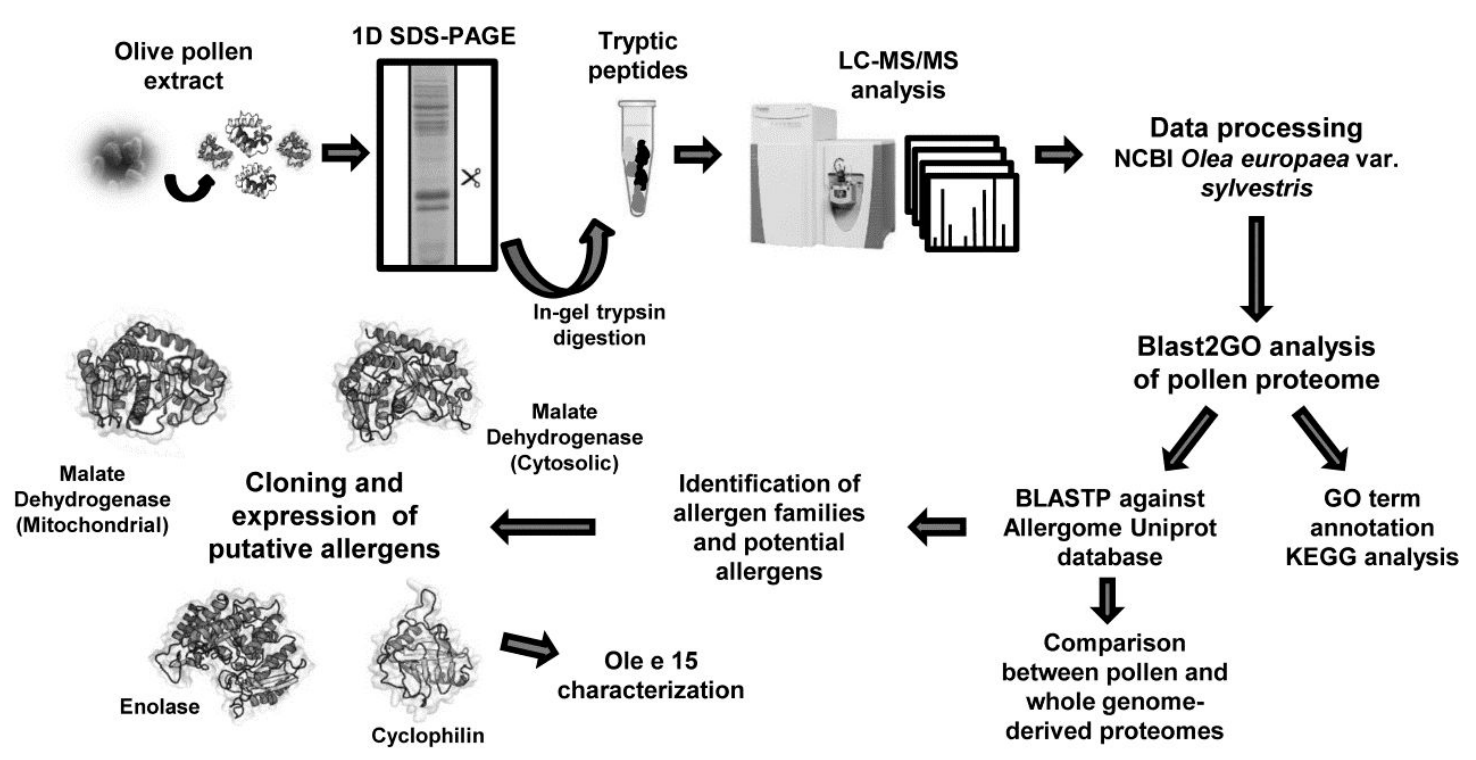

Figure 1. Workflow of the study to delineate the olive pollen proteome and its allergenome, and describe Ole e 15 as a new relevant allergen implicated in cross-reactivity. 3D-models of the proteins were created using 1sev.1.A, 5.nue.1.B, 1xsx.1.A and 2mc9 PDB structures for malate dehydrogenase (mitochondrial), malate dehydrogenase (cytosolic), enolase and cyclophilin, respectively.

First, three olive pollen protein extracts replicates were fractionated by SDS-PAGE and protein bands in-gel trypsin digested. Then, the extracted peptides were analyzed by LCMS/MS with a Q-exactive mass spectrometer. Next, the quality of the MS results was investigated calculating the mean and SD of the mass error in ppm in the three replicates. For replicate 1 the mean \pm SD mass error was 0.66 and 1.84 , for replicate 2 was 2.39 and 1.64 , and for replicate 3 was 2.41 and 1.68. As the data showed a good quality, we proceeded to subsequent analyses.

From the three biological replicates, a total of 1,907 different proteins were identified (Table S3 and Figure S1). Protein sequences identified from olive pollen were functionally mapped and 
A

\begin{tabular}{|c|c|}
\hline & Number of sequences \\
\hline Total Sequences analyzed & 1,907 \\
\hline With Protein Blast Hits & 1,907 \\
\hline With Mapping & 1,787 \\
\hline With InterProScan & 1,568 \\
\hline With Annotation & 1,738 \\
\hline SignalP 4.1 & 239 \\
\hline
\end{tabular}

B

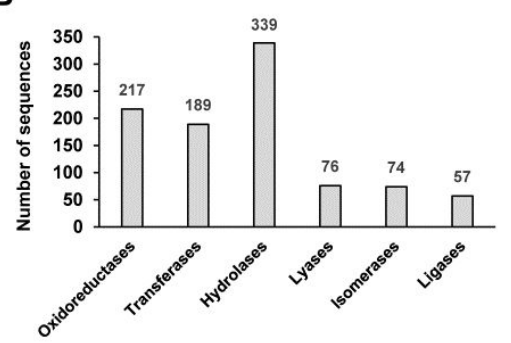

D

\begin{tabular}{|c|c|c|}
\hline & $\begin{array}{c}\text { Wild olive } \\
\text { genome-derived } \\
\text { proteome }\end{array}$ & $\begin{array}{c}\text { Pollen proteome } \\
\text { (LC-MS/MS } \\
\text { analysis) }\end{array}$ \\
\hline $\begin{array}{c}\text { Number of } \\
\text { Allergome hits }\end{array}$ & 3,469 & 363 \\
\hline $\begin{array}{c}\text { Potential allergens } \\
\text { (applied criteria) }\end{array}$ & 2,446 & 203 \\
\hline $\begin{array}{c}\text { Potential allergen } \\
\text { families (applied } \\
\text { criteria) }\end{array}$ & 76 & 47 \\
\hline
\end{tabular}

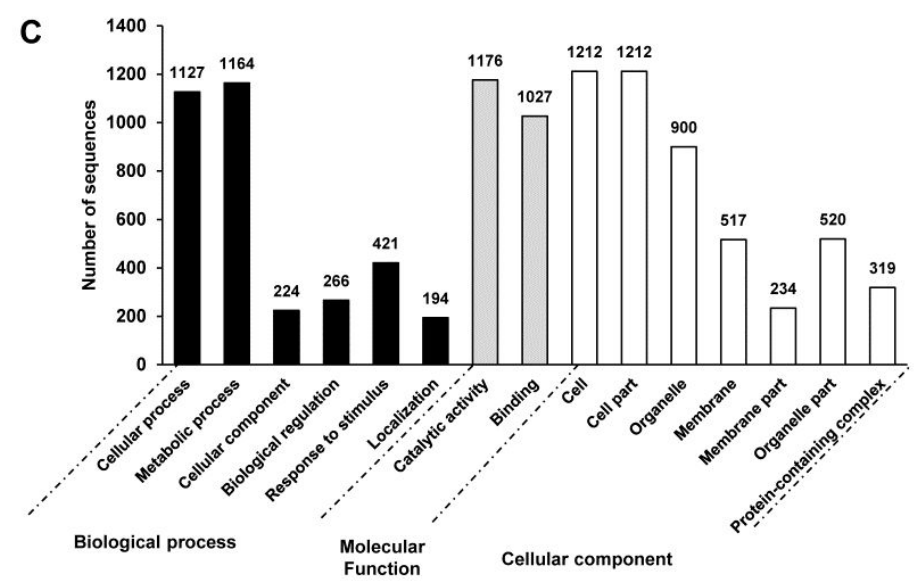

Figure 2. Classification of the olive pollen proteome according to the annotated GO terms after Blast2GO analysis and identification of potential allergens. (a) Summary table with the general results after Blast2GO and SignalP 4.1 bioinformatic analysis. (b) Biological process, molecular function and cellular component GO term distribution on the level 2 of complexity. (c) Number of proteins of each enzyme class after GO-Enzyme Code mapping. (d) Table summarizing the main results after protein Blast against the Allergome database and application of criteria for the identification of potential allergens and allergen families.

Cellular component terms revealed that identified olive pollen proteins were widely distributed among the cell, with more than 500 proteins associated to membranes. Metabolic processes were the most abundant in biological process terms (Figure $2 \mathrm{~b}$ and Figure S2). Regarding molecular function, cyclic compound, ion and protein binding activities, and hydrolase activity, were highly represented (Figure S2). To be noticed, 1,176 out of the 1,907 identified proteins displayed catalytic activity (61.7\%), being hydrolases, oxidoreductases and transferases the most abundant enzyme classes found (Figure 2c and Table S4). KEGG pathway analysis showed that 7 out of the 10 most enriched pathways were related to metabolism (Figure 
S3a). To analyze the enrichment of certain GO terms in pollen, a Fisher's exact test was performed comparing the annotated GO terms of the pollen proteome with that of the genomederived proteome (Figure S3b and Table S4). GO terms related to salt stress, salt absorption and amino acid and sugar metabolism were over-represented in pollen. Moreover, 239 (12.5\%) of the identified pollen proteins were predicted as secreted by SignalP 4.1 (Table S5).

\section{Olive pollen allergenome delineation reveals the presence of 47 allergen families}

Next, the delineation of the olive pollen allergenome was investigated to identify putative allergens in this allergenic source with high clinical relevance (17). Firstly, the whole wild olive genome-predicted proteome and the olive pollen identified proteome were locally blasted against the Allergome database. A total of 3,469 and 363 hits were retrieved, respectively. Then, a set of criteria (see Methods) were manually applied to filter the results and identify potential allergens. Of the total of 2,446 potential allergens belonging to 76 different allergen families in the wild olive predicted-proteome, 203 proteins belonging to 47 allergen families were present in pollen (Figure 2d, Table 1, Table S6 and Table S7). Among them, 10 families previously reported as actual olive pollen allergens were observed (17). Moreover, sixty of these proteins (29.6\%) were predicted to be secreted by SignalP 4.1 (Table S5), which is one the most common features of allergenic proteins. 
Table 1. Olive pollen allergenome definition by in-depth proteomics and bioinformatics using the Allergome database.

\begin{tabular}{|c|c|c|c|c|c|c|c|c|c|c|}
\hline $\begin{array}{ll}6 & \\
7 & \\
8 & \text { Accession } \\
9 & \text { Number* } \\
10 & \\
11 & \end{array}$ & Protein name & $\begin{array}{c}\text { Homologous } \\
\text { allergen in } \\
\text { the IUIS } \\
\text { database }\end{array}$ & $\begin{array}{c}\text { Uniprot } \\
\text { Code }\end{array}$ & Organism & $\begin{array}{c}\text { Amino acid } \\
\text { similarity/identity } \\
(\%)\end{array}$ & E-value & $\begin{array}{c}\text { Allergen } \\
\text { family name }\end{array}$ & $\begin{array}{l}\text { All } \\
\text { Fam } \\
\text { Code }\end{array}$ & $\begin{array}{c}\text { Route of } \\
\text { sensitization }\end{array}$ & $\begin{array}{c}\text { Total potential } \\
\text { olive pollen } \\
\text { allergens } \\
\text { identified in the } \\
\text { family**** }\end{array}$ \\
\hline $\begin{array}{l}12 \\
\text { 13P_022892128.1 } \\
14\end{array}$ & Major pollen allergen. Ole e 1 -like & Ole e 1 & P19963 & $\begin{array}{c}\text { O. europaea } \\
\text { (Cultivated olive) }\end{array}$ & $99 / 95$ & 7.4E-107 & Ole e 1-like & AF087 & Inhalation & 17 \\
\hline $\begin{array}{l}15 \\
1 \text { YP_022882512.1 } \\
17\end{array}$ & Profilin-3 & Ole e 2 & A4GDU2 & $\begin{array}{c}\text { O. europaea } \\
\text { (Cultivated olive) }\end{array}$ & $100 / 98$ & 0 & Profilin & AF051 & Inhalation & 4 \\
\hline $\begin{array}{l}18 \\
\text { 18甲_022850789.1 } \\
20\end{array}$ & Polcalcin & Ole e 3 & 081092 & $\begin{array}{c}\text { O. europaea } \\
\text { (Cultivated olive) }\end{array}$ & $100 / 98$ & 0 & EF-Hand & AF007 & Inhalation & 5 \\
\hline $\begin{array}{l}21 \\
22 \text { ¿2022849259.1 } \\
23\end{array}$ & Superoxide dismutase $[\mathrm{Cu}-\mathrm{Zn}] 2$ & Ole e 5 & Q8L5E0 & $\begin{array}{c}\text { O. euroapea } \\
\text { (Cultivated olive) }\end{array}$ & $100 / 100$ & 0 & $\begin{array}{l}\text { Cu/Zn Superoxide } \\
\text { Dismutase }\end{array}$ & AF020 & Inhalation & 2 \\
\hline $\begin{array}{l}24 \\
2 \Varangle P_{-022896469.1} \\
26\end{array}$ & Non-specific lipid transfer protein & Ole e 7 & $(18)^{* \star}$ & $\begin{array}{c}\text { O. europaea } \\
\text { (Cultivated olive) }\end{array}$ & $81 / 74$ & - & Prolamin & AF050 & Inhalation & 3 \\
\hline $\begin{array}{l}27 \\
288 \_022899478.1 \\
29\end{array}$ & $\begin{array}{l}\text { Glucan endo-1,3-beta-D- } \\
\text { glucosidase }\end{array}$ & Ole e 9 & D8VPP5 & $\begin{array}{c}\text { O. europaea } \\
\text { (Cultivated olive) }\end{array}$ & $100 / 99$ & 0 & $\begin{array}{c}\text { Beta-1,3- } \\
\text { glucanase and } \mathrm{X} 8 \\
\text { domain }\end{array}$ & AF063 & Inhalation & 7 \\
\hline $\begin{array}{l}30 \\
\text { 3XP_022888359.1 } \\
32\end{array}$ & Pectinesterase 1 & Ole e 11 & A4GDU2 & $\begin{array}{c}\text { O. europaea } \\
\text { (Cultivated olive) }\end{array}$ & $99 / 99$ & 0 & $\begin{array}{c}\text { Pectin } \\
\text { methylesterase }\end{array}$ & AF026 & Inhalation & 9 \\
\hline $\begin{array}{l}33 \\
34 P_{-} 022894573.1 \\
35\end{array}$ & Isoflavone reductase-like protein & Ole e 12 & E1U332 & $\begin{array}{c}\text { O. europaea } \\
\text { (Cultivated olive) }\end{array}$ & $100 / 99$ & 0 & $\begin{array}{l}\text { Isoflavone } \\
\text { reductase }\end{array}$ & AF123 & Inhalation & 5 \\
\hline $\begin{array}{l}36 \\
\text { 3XP_022859834.1 } \\
38\end{array}$ & Protein P21-like & Act $d 2$ & L7TRX2 & $\begin{array}{c}\text { A.deliciosa } \\
\text { (Kiwifruit) }\end{array}$ & $74 / 57$ & 1.69E-97 & $\begin{array}{c}\text { Thaumatin-like } \\
\text { Protein (Ole e 13) }\end{array}$ & AF060 & Ingestion & 1 \\
\hline
\end{tabular}




\begin{tabular}{|c|c|c|c|c|c|c|c|c|c|c|}
\hline $\begin{array}{l}4 \\
\text { 5XP_022872535.1 } \\
6\end{array}$ & Exopolygalacturonase-like & Pla a 2 & Q6H9K0 & $\begin{array}{c}\text { P. } x \text { acerifolia } \\
\text { (London planetree) }\end{array}$ & $73 / 51$ & $2.15 \mathrm{E}-142$ & $\begin{array}{l}\text { Polygalacturonase } \\
\text { (Ole e 14) }\end{array}$ & AF057 & Inhalation & 4 \\
\hline $\begin{array}{l}7 \\
8 X P \_022846733.1 \\
9\end{array}$ & Enolase 2 & Hev b 9 & Q9LEI9 & $\begin{array}{c}\text { H. brasiliensis } \\
\text { (Latex) }\end{array}$ & $96 / 89$ & 0 & Enolase & AF031 & Contact & 4 \\
\hline $\begin{array}{l}10 \\
12 \\
12\end{array}$ & Glutathione S-transferase L3-like & Bet $v 8$ & A0A096XCA4 & $\begin{array}{l}\text { B. verrucosa } \\
\text { (Birch) }\end{array}$ & $82 / 69$ & 3.76E-121 & $\begin{array}{l}\text { Gutathione S- } \\
\text { transferase }\end{array}$ & AF010 & Inhalation & 1 \\
\hline $\begin{array}{l}13 \\
1 P_{-} 022880502.1\end{array}$ & Cysteine proteinase inhibitor 1 -like & Act d 4 & Q6TPK4 & $\begin{array}{l}\text { A. deliciosa } \\
\text { (Kiwifruit) }\end{array}$ & $77 / 51$ & 9.97E-34 & Cystatin & AF005 & Ingestion & 1 \\
\hline $\begin{array}{l}16 \\
16 \\
18\end{array}$ & Probable cysteine protease RD19B & Act d 1 & A5HIJ3 & $\begin{array}{l}\text { A. deliciosa } \\
\text { (Kiwifruit) }\end{array}$ & $91 / 80$ & 0 & $\begin{array}{c}\text { Papain-like } \\
\text { cysteine protease }\end{array}$ & AF030 & Ingestion & 9 \\
\hline $\begin{array}{l}19 \\
20 P \_022857847.1 \\
21 \\
22\end{array}$ & $\begin{array}{l}5- \\
\text { Methyltetrahydropteroyltriglutamate- } \\
\text { homocysteine methyltransferase }\end{array}$ & Sal k 3 & C1KEU0 & $\begin{array}{c}\text { S. kali } \\
\text { (Russian thistle) }\end{array}$ & $95 / 89$ & 0 & $\begin{array}{l}\text { Cobalamin- } \\
\text { independent } \\
\text { methionine } \\
\text { synthase }\end{array}$ & AF152 & Inhalation & 7 \\
\hline $\begin{array}{l}23 \\
24-022854141.1 \\
25\end{array}$ & Probable pectate lyase P59 & Art $\vee 6$ & A0PJ16 & $\begin{array}{l}\text { A. vulgaris } \\
\text { (Mugwort) }\end{array}$ & $65 / 49$ & 1.63E-40 & Pectate lyase & AF073 & Inhalation & 3 \\
\hline $\begin{array}{l}26 \\
28 \\
28\end{array}$ & $\begin{array}{l}\text { Superoxide dismutase [Mn], } \\
\text { mitochondrial-like isoform X2 }\end{array}$ & Hev b 10 & P35017 & $\begin{array}{c}\text { H. brasiliensis } \\
\text { (Latex) }\end{array}$ & $90 / 83$ & $7.5 \mathrm{E}-145$ & $\begin{array}{l}\text { Fe/Mn superoxide } \\
\text { dismutase }\end{array}$ & AF019 & Contact & 2 \\
\hline $\begin{array}{l}29 \\
38-022880113.1 \\
31\end{array}$ & $\begin{array}{c}\mathrm{NAD}(\mathrm{P}) \mathrm{H} \text { dehydrogenase (quinone) } \\
\mathrm{FQR} 1 \text { isoform } \mathrm{X} 1\end{array}$ & Alt a 7 & P42058 & $\begin{array}{l}\text { A. alternata } \\
\text { (Alternaria) }\end{array}$ & $71 / 55$ & $1.68 \mathrm{E}-56$ & $\begin{array}{l}\text { NADPH- } \\
\text { dependent FMN } \\
\text { reductase }\end{array}$ & AF053 & Inhalation & 7 \\
\hline $\begin{array}{l}32 \\
3 \% \text { ßP_022853324.1 } \\
34\end{array}$ & $\begin{array}{l}\text { 60S acidic ribosomal protein P2B- } \\
\text { like }\end{array}$ & Pru du 5 & Q8H2B9 & $\begin{array}{l}\text { P. dulcis } \\
\text { (Almond) }\end{array}$ & $95 / 85$ & 1.94E-31 & $\begin{array}{l}60 S \text { acidic } \\
\text { ribosomal protein }\end{array}$ & AF070 & Ingestion & 1 \\
\hline $\begin{array}{l}35 \\
3 \text { 好_022854082.1 } \\
37\end{array}$ & Luminal-binding protein 5-like & Cor a 10 & Q9FSY7 & $\begin{array}{c}\text { C. avellana } \\
\text { (Common Hazel) }\end{array}$ & $97 / 92$ & 0 & $\begin{array}{l}\text { Heat shock } \\
\text { protein HSP70 }\end{array}$ & AF002 & Ingestion & 22 \\
\hline $\begin{array}{l}38 P \_022897418.1 \\
39\end{array}$ & Peroxiredoxin-2 & Mala f 3 & P56578 & M. furfur & $60 / 45$ & $5.9 \mathrm{E}-44$ & Redoxin & AF131 & Inhalation & 5 \\
\hline
\end{tabular}




\begin{tabular}{|c|c|c|c|c|c|c|c|c|c|c|}
\hline $\begin{array}{l}4 \\
\text { 5XP_022855565.1 } \\
6\end{array}$ & $\begin{array}{c}\text { Soluble inorganic pyrophosphatase } \\
6 \text {, chloroplastic }\end{array}$ & Der f 32 & A0A088SCP3 & $\begin{array}{c}\text { D. farinae } \\
\text { (Human dust mite) }\end{array}$ & $67 / 47$ & $2.04 \mathrm{E}-74$ & $\begin{array}{c}\text { Inorganic } \\
\text { pyrophosphatase }\end{array}$ & AF205 & Inhalation & 1 \\
\hline $\begin{array}{l}7 \\
8 X P \_022885711.1 \\
9\end{array}$ & Thioredoxin H-type & Tri a 25 & Q9LDX4 & $\begin{array}{c}\text { T. aestivum } \\
\text { (Wheat) }\end{array}$ & $76 / 57$ & $9.12 \mathrm{E}-47$ & Thioredoxin & AF023 & Ingestion & 5 \\
\hline $\begin{array}{l}10 \\
1 X P \_022875019.1 \\
12 \\
12\end{array}$ & Actin-depolymerizing factor -like & Der f 31 & A0A088SAY1 & $\begin{array}{c}\text { D. farinae } \\
\text { (Human dust mite) }\end{array}$ & $59 / 38$ & $1.32 \mathrm{E}-23$ & $\begin{array}{c}\text { Actin- } \\
\text { depolymerizing } \\
\text { factor-homolog }\end{array}$ & AF201 & Inhalation & 6 \\
\hline $\begin{array}{l}13 \\
15 \\
15\end{array}$ & $\begin{array}{l}\text { Ferritin-2, chloroplastic-like isoform } \\
\text { X2 }\end{array}$ & Der $f 30$ & L7UZ91 & $\begin{array}{c}\text { D. farinae } \\
\text { (Human dust mite) }\end{array}$ & $56 / 39$ & 1.7E-19 & Ferritin & AF198 & Inhalation & 1 \\
\hline $\begin{array}{l}16 \\
18\end{array}$ & $\begin{array}{l}\text { Aldehyde dehydrogenase family } 2 \\
\text { member B7, mitochondrial-like }\end{array}$ & Cla h 10 & P40108 & C. herbarum & $68 / 56$ & $2.04 \mathrm{E}-74$ & $\begin{array}{c}\text { Aldehyde } \\
\text { dehydrogenase }\end{array}$ & AF040 & Inhalation & 5 \\
\hline $\begin{array}{l}19 \\
2 \mathrm{XP} \_022867276.1 \\
21 \\
22\end{array}$ & Aspartic proteinase-like & Aed a 11 & Q03168 & $\begin{array}{c}\text { A. aegypti } \\
\text { (Yellow fever } \\
\text { mosquito) }\end{array}$ & $72 / 44$ & $1.28 \mathrm{E}-84$ & $\begin{array}{l}\text { Eukaryotic } \\
\text { Aspartyl Protease }\end{array}$ & AF004 & Contact & 2 \\
\hline $\begin{array}{l}23 \\
24-022896740.1 \\
25\end{array}$ & $\begin{array}{l}\text { Peptidyl-prolyl cis-trans } \\
\text { isomerase } 1\end{array}$ & Hev b Сyp & F8RW92 & $\begin{array}{l}\text { H. brasiliensis } \\
\text { (Latex) }\end{array}$ & $97 / 91$ & $1.58 \mathrm{E}-109$ & Cyclophilin & AF038 & Inhalation/Contact & 7 \\
\hline $\begin{array}{l}26 P_{-} 022855094.1 \\
27\end{array}$ & Ribosomal protein L3-2 & Asp $\mathrm{f} 23$ & BOXSL2 & A. fumigatus & $73 / 69$ & $1.9 \mathrm{E}-105$ & $\begin{array}{c}\text { Ribosomal Protein } \\
\text { L3 }\end{array}$ & AF058 & Inhalation & 1 \\
\hline $\begin{array}{l}28 \\
2 X P \_022895071.1 \\
30\end{array}$ & $\begin{array}{l}\text { Glyceraldehyde-3-phosphate } \\
\text { dehydrogenase, cytosolic }\end{array}$ & Tri a 34 & $\mathrm{C} 7 \mathrm{C} 4 \mathrm{X} 1$ & $\begin{array}{l}\text { T. aestivum } \\
\text { (Wheat) }\end{array}$ & $95 / 87$ & $5.9 \mathrm{E}-101$ & $\begin{array}{c}\text { Glyceraldehyde 3- } \\
\text { phosphate } \\
\text { dehydrogenase }\end{array}$ & AF184 & Inhalation & 7 \\
\hline $\begin{array}{l}31 \\
3 \times 2 P \_022882931.1 \\
33\end{array}$ & Subtilisin-like protease SBT4.15 & Cuc m 1 & Q39547 & $\begin{array}{l}\text { C. melo } \\
\text { (Melon) }\end{array}$ & $64 / 47$ & 0 & $\begin{array}{l}\text { Subtilisin-like } \\
\text { serine protease }\end{array}$ & AF021 & Ingestion & 5 \\
\hline $\begin{array}{l}34 \\
3 \times P_{1} 022862800.1 \\
36\end{array}$ & $\begin{array}{l}\text { Malate dehydrogenase, } \\
\text { mitochondrial }\end{array}$ & MDH cit I & P17783 & $\begin{array}{c}\text { C. lanatus } \\
\text { (Watermelon) }\end{array}$ & $97 / 86$ & 0 & \multirow[t]{2}{*}{$\begin{array}{l}\text { Lactate/Malate } \\
\text { dehydrogenase }\end{array}$} & \multirow[t]{2}{*}{ AF014 } & \multirow[t]{2}{*}{ Ingestion/Inhalation } & \multirow[t]{2}{*}{9} \\
\hline $\begin{array}{l}37 P_{2} 022874613.1 \\
38\end{array}$ & Malate dehydrogenase, cytosolic & - & $(37)^{\star \star \star}$ & F. excelsior & - & - & & & & \\
\hline
\end{tabular}




\begin{tabular}{|c|c|c|c|c|c|c|c|c|c|c|}
\hline $\begin{array}{l}4 \\
5\end{array}$ & & & & (Ash) & & & & & & \\
\hline $\begin{array}{l}6 \\
7 \\
8\end{array}$ & $\begin{array}{c}\text { Probable serine/threonine-protein } \\
\text { kinase PBL23 }\end{array}$ & Sal k 2 & Q8L5K9 & $\begin{array}{c}\text { S. kali } \\
\text { (Russian thistle) }\end{array}$ & $60 / 36$ & $3.73 E-53$ & Protein kinase & AF017 & Inhalation & 2 \\
\hline $\begin{array}{l}9 \\
\text { XP_022876859.1 } \\
11\end{array}$ & $\begin{array}{l}\text { Reactive Intermediate Deaminase } \\
\text { A, chloroplastic-like }\end{array}$ & Der $\mathrm{f} 34$ & A0A1J1DL12 & $\begin{array}{c}\text { D. farinae } \\
\text { (Human dust mite) }\end{array}$ & $58 / 30$ & $8.23 \mathrm{E}-18$ & $\begin{array}{l}\text { RidA/YjgF/L-PSP } \\
\text { family }\end{array}$ & AF213 & Inhalation & 1 \\
\hline $\begin{array}{l}12 \\
13 \text { P_022847291.1 } \\
14\end{array}$ & Stress-related protein-like & Hev b 3 & F8SKC3 & $\begin{array}{c}\text { H. brasiliensis } \\
\text { (Latex) }\end{array}$ & $66 / 50$ & $3.67 E-58$ & $\begin{array}{l}\text { Rubber elongation } \\
\text { factor }\end{array}$ & AF125 & Contact & 2 \\
\hline $\begin{array}{l}15 \\
150-022891594.1 \\
17\end{array}$ & Aldehyde oxidase GLOX-like & Art an 7 & $\mathrm{~A} 0 \mathrm{~A} 2 \mathrm{H} 4 \mathrm{HHY} 6$ & $\begin{array}{c}\text { A. annua } \\
\text { (Sweet wormwood) }\end{array}$ & $57 / 39$ & $2.27 \mathrm{E}-132$ & Galactose oxidase & - & Inhalation & 1 \\
\hline $\begin{array}{l}188 \_022843917.1 \\
19\end{array}$ & Cytochrome c & Cur I 3 & Q96VP3 & C. lunata & $78 / 56$ & 1.77E-44 & Cytochrome c & AF006 & Inhalation & 2 \\
\hline 21 & Elongation factor 1-delta & Pen c 24 & Q69BZ7 & P. citrinum & $78 / 40$ & $3.83 \mathrm{E}-24$ & $\begin{array}{c}\text { Eukaryotic } \\
\text { elongation factor } 1\end{array}$ & AF011 & Inhalation & 2 \\
\hline $\begin{array}{l}22 \\
23 \text { ¿ł_022844073.1 } \\
24\end{array}$ & Serpin-ZX-like & Tri a 33 & P93692 & $\begin{array}{l}\text { T. aestivum } \\
\text { (Wheat) }\end{array}$ & $72 / 55$ & $1.34 \mathrm{E}-144$ & $\begin{array}{l}\text { Serpin serine } \\
\text { protease inhibitor }\end{array}$ & AF018 & Inhalation & 1 \\
\hline $\begin{array}{l}25 \\
2 \% 8 \_022891403.1 \\
27\end{array}$ & $\begin{array}{l}\text { 3-oxoacyl-[acyl-carrier-protein] } \\
\text { reductase } 4 \text {-like }\end{array}$ & Alt a 8 & P0COY4 & $\begin{array}{l}\text { A. alternata } \\
\text { (Alternaria) }\end{array}$ & $55 / 32$ & $1.82 \mathrm{E}-30$ & $\begin{array}{l}\text { Short chain } \\
\text { dehydrogenase }\end{array}$ & AF028 & Inhalation & 3 \\
\hline $\begin{array}{l}28 \\
2 \% \_022890941.1 \\
30\end{array}$ & $\begin{array}{l}\text { Triosephosphate isomerase, } \\
\text { cytosolic-like, partial }\end{array}$ & Tri a 31 & Q9FS79 & $\begin{array}{l}\text { T. aestivum } \\
\text { (Wheat) }\end{array}$ & $91 / 80$ & 0 & $\begin{array}{l}\text { Triosephosphate } \\
\text { isomerase }\end{array}$ & AF032 & Inhalation & 4 \\
\hline $\begin{array}{l}31 \\
3 \nVdash \_022863167.1 \\
33\end{array}$ & $\begin{array}{l}\text { Vicilin-like seed storage protein } \\
\text { At2g28490, partial }\end{array}$ & Coc $n 1$ & A0A0S3B0K0 & $\begin{array}{c}\text { C. nucifera } \\
\text { (Coconut tree) }\end{array}$ & $70 / 52$ & $2.03 E-108$ & Cupin & AF045 & Ingestion & 1 \\
\hline $\begin{array}{l}34 \\
3 \nvdash P_{0} 022856299.1 \\
36\end{array}$ & Major allergen Pru ar 1-like & Pru ar 1 & 050001 & $\begin{array}{l}\text { P. armeniaca } \\
\text { (Apricot tree) }\end{array}$ & $79 / 67$ & $1.46 \mathrm{E}-63$ & Bet $v 1$ family & AF069 & Ingestion & 1 \\
\hline $\begin{array}{l}37 \text { _ } 022861507.1 \\
38-0228\end{array}$ & $\begin{array}{l}\text { Eukaryotic translation initiation } \\
\text { factor } 3 \text { subunit I-like }\end{array}$ & For $\mathrm{t} 2$ & B2ZPG7 & F. taiwana & $68 / 48$ & 7.37E-112 & WD-40 Repeat & AF142 & Contact & 1 \\
\hline
\end{tabular}




\begin{tabular}{|c|c|c|c|c|c|c|c|c|c|c|}
\hline \begin{tabular}{|l}
4 \\
5 \\
\end{tabular} & & & & (Biting midge) & & & & & & \\
\hline $\begin{array}{l}6 \\
7 P_{-} 022899451.1 \\
8\end{array}$ & $\begin{array}{l}\text { Fructose-bisphosphate aldolase, } \\
\text { cytoplasmic isozyme-like }\end{array}$ & Sal s 3 & $\mathrm{COH} 9 \mathrm{I} 1$ & $\begin{array}{c}\text { S. salar } \\
\text { (Atlantic salmon) }\end{array}$ & $74 / 60$ & $2.48 \mathrm{E}-145$ & $\begin{array}{c}\text { Fructose } \\
\text { bisphosphate } \\
\text { aldolase class I }\end{array}$ & AF197 & Ingestion & 5 \\
\hline $\begin{array}{l}9 \\
P \ngtr \_022874390.1 \\
11\end{array}$ & $\begin{array}{c}\text { Acid beta-fructofuranosidase } 2 \text {, } \\
\text { vacuolar-like }\end{array}$ & Sola I 2 & Q8RVW4 & $\begin{array}{l}\text { S. Iycopersicum } \\
\text { (Tomato) }\end{array}$ & $80 / 60$ & 0 & $\begin{array}{c}\text { Glycoside } \\
\text { hydrolase family } \\
32\end{array}$ & AF052 & Ingestion & 3 \\
\hline $\begin{array}{l}12 \\
13 \\
14\end{array}$ & Calnexin homolog 1-like & Pen ch 31 & Q2TL59 & P. chrysogenum & $67 / 44$ & $\begin{array}{l}5.75998 \mathrm{E}- \\
93\end{array}$ & Calreticulin & AF055 & Inhalation & 6 \\
\hline
\end{tabular}

MS/MS spectra for the 5 proteins with single peptide entries in the three biological replicates (Glutathione S-transferase L3-like, Isoflavone reductase-like protein, Peptidyl-prolyl cis-trans isomerase CYP21-like, Pollen allergen Che a 1-like and Superoxide dismutase [Cu-Zn] 2-like isoform X3) are shown in Figure S4.

* NCBI Accession numbers of the Olea europaea var. sylvestris proteins sharing the highest sequence identity with Allergome allergens of the indicated allergen families (see also Table S7).

** Ole e 7 sequence has been recently published and is not yet deposited in Uniprot (18).

*** Cytosolic malate dehydrogenase from ash (F. excelsior) pollen, a member of the Oleaceae family, was suggested as potential allergen in (39).

**** Isoallergens, allergen variants and different allergenic proteins belonging to the same family (see also Table S6 and Table S7 for a full description of the allergens identified).

\section{One of the four selected allergen candidates, cyclophilin from olive pollen, shows significant IgE reactivity}

After proteomic analysis, four potential allergens among the 47 allergen families identified from olive pollen (cyclophilin, enolase, and cytosolic and mitochondrial malate dehydrogenases) were selected for validation to assess their IgE reactivity. Three different cDNA clones from cyclophilin and one cDNA clone for the rest of the proteins were obtained (Table S8). All proteins shared $>95 \%$ sequence identity with predicted proteins in the Olea europaea var. sylvestris database. 
We then proceeded to express and purify the potential allergens to analyze their $\operatorname{IgE}$ seroreactivity. The predicted mature forms of the proteins were expressed in E. coli as $\mathrm{His}_{6}{ }^{-}$ tagged N-terminal proteins. A purity $>95 \%$ for all proteins was achieved (Figure 3a). Proteins were subsequently analyzed by ELISA using sera from olive pollen allergic patients from two populations from Spain -Madrid and Córdoba-, and five non-atopic individuals.

A

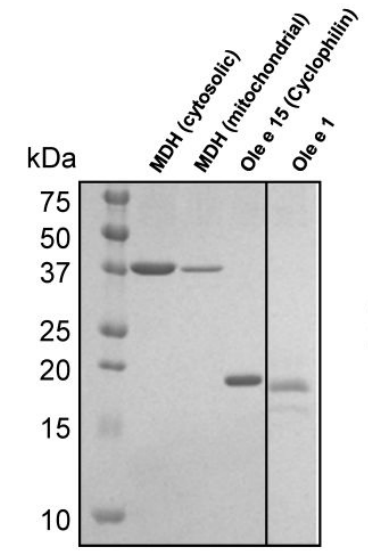

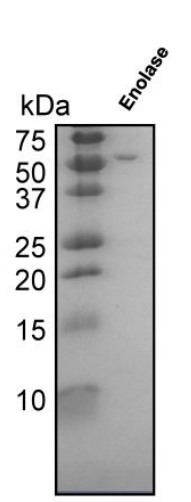

B
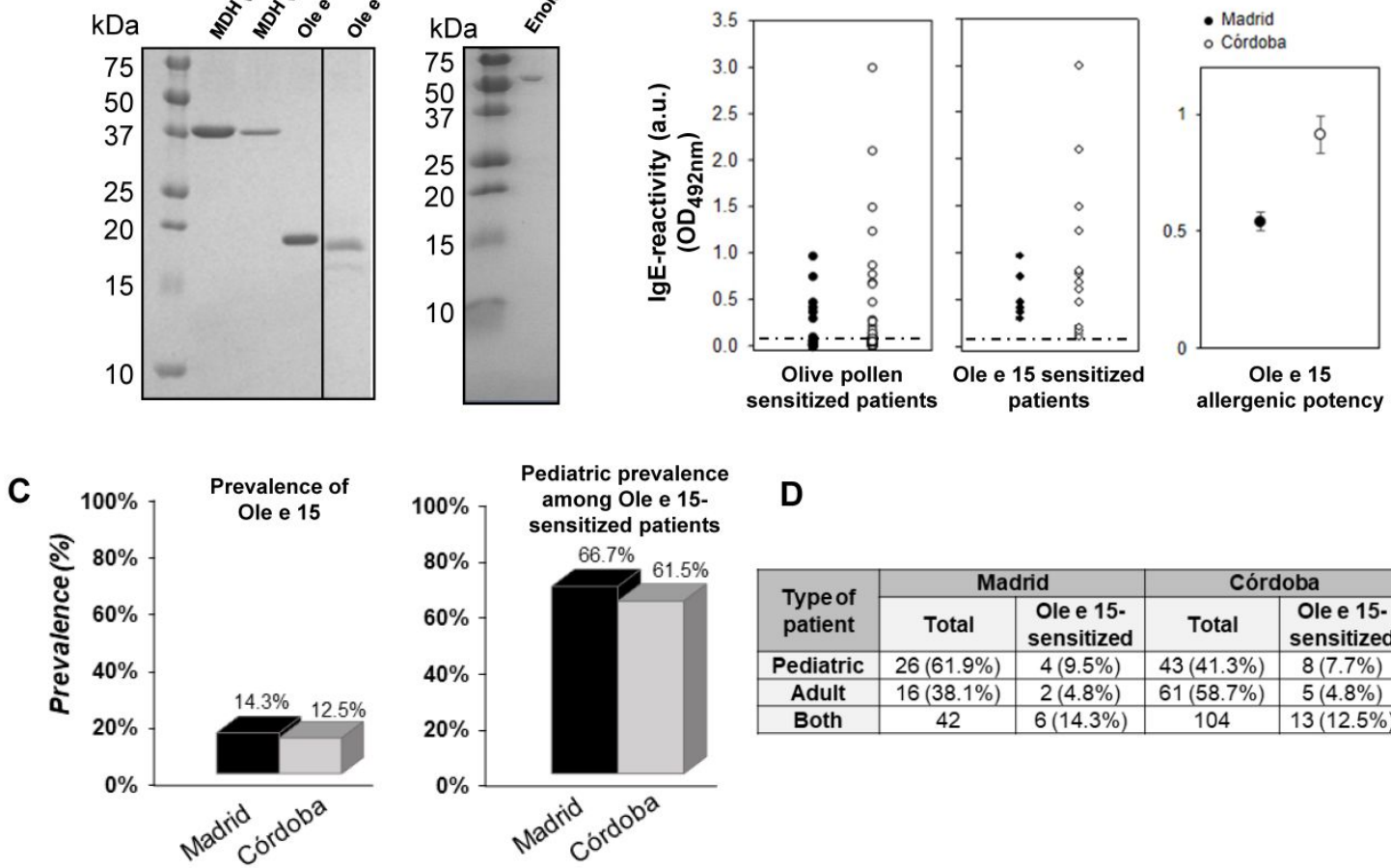

D

\begin{tabular}{|c|c|c|c|c|}
\hline \multirow{2}{*}{$\begin{array}{c}\text { Type of } \\
\text { patient }\end{array}$} & \multicolumn{2}{|c|}{ Madrid } & \multicolumn{2}{c|}{ Córdoba } \\
\cline { 2 - 5 } & Total & $\begin{array}{c}\text { Ole e 15- } \\
\text { sensitized }\end{array}$ & Total & $\begin{array}{c}\text { Ole e 15- } \\
\text { sensitized }\end{array}$ \\
\hline Pediatric & $26(61.9 \%)$ & $4(9.5 \%)$ & $43(41.3 \%)$ & $8(7.7 \%)$ \\
\hline Adult & $16(38.1 \%)$ & $2(4.8 \%)$ & $61(58.7 \%)$ & $5(4.8 \%)$ \\
\hline Both & 42 & $6(14.3 \%)$ & 104 & $13(12.5 \%)$ \\
\hline
\end{tabular}

Figure 3. Analysis of the purified recombinant proteins and IgE-reactivity to rOle e 15 . (a) Analysis of $0.5 \mu \mathrm{g}$ purified recombinant proteins by Coomassie Blue staining after 15\% SDSPAGE under reducing conditions. Purified natural Ole e 1 was used as control. (b) Dot plots representing the strength of the IgE reactivity to rOle e 15 . The dotted line indicates the response threshold (O.D. values>0.1). (c) Bar graphs representing the prevalence of Ole e 15sensitized patients in both populations (left) and the pediatric prevalence among rOle e 15sensitized patients (right). (d) Prevalence data are also displayed.

Only olive pollen cyclophilin showed IgE recognition, and thus, was named Ole e 15 according to WHO/IUIS (Figure 3b). No significant differences in the recognition frequency were observed between both patients' populations, with prevalence values of $14.3 \%$ and $12.5 \%$ in Madrid and Córdoba, respectively (Figure 3c).

Most of the allergic patients showed high IgE levels against the allergen, especially in the population from Córdoba. Furthermore, we observed a higher number of pediatric patients than 
adults among the Ole e 15 sensitized patients. In total, 8 out of the 13 allergic patients detected in Córdoba (61.5\%) and 4 out of the 6 allergic patients detected in Madrid (66.7\%) were pediatric (Figure 3c).

\section{Recombinant Ole e 15 shows the typical folding, shares high sequence identity with other plant, animal and fungal cyclophilins and is involved in cross-reactivity}

Next, we proceeded to further physicochemically and immunologically characterize this new olive pollen allergen. To this end, we initially assessed by mass spectrometry and $\mathrm{CD}$ its correct molecular mass and folding, respectively. First, an experimental molecular mass of 19,408 Da was observed for His $_{6}$-tagged rOle e 15, in agreement with the expected theoretical molecular mass without the initial Met (19,391 Da), which is processed when proteins are produced in bacteria. Far-UV CD spectra and percentages of secondary structure of rOle e 15 were in agreement with those described for other cyclophilins. In addition, thermal denaturation experiments for rOle e 15 revealed a $\mathrm{Tm}$ of $47^{\circ} \mathrm{C}$, being unable to recover its structure cooling afterwards (Figure S5).

Ole e 15 amino acid sequence alignment with other plant, animal and fungal allergenic cyclophilins showed a high degree of conservation, with identities of $94 \%, 68 \%$ and $65 \%$ with Sola 1 5, Der f 29 and Mala s 6, respectively (Figure S6). Molecular modelling revealed a predicted structure with the typical plant cyclophilin folding (Figure 4a-c and Figure S6), consisting of a $\beta$-barrel of seven anti-parallel strands, two surrounding $\alpha$-helices and a characteristic stretch of seven amino acids called the divergent loop, which is mainly present in plants (40). Moreover, the predicted amino acids defining the active surface in the cyclophilin family were present (Figure 4a) (41). Its theoretical surface electrostatic potential was mainly basic, but showed a neutral potential in the putative active surface region (Figure $4 \mathrm{~b}$ ). In addition, other cyclophilins were modelled and compared to Ole e 15 (Figure 4c), showing an almost identical folding among them except for the divergent loop. 


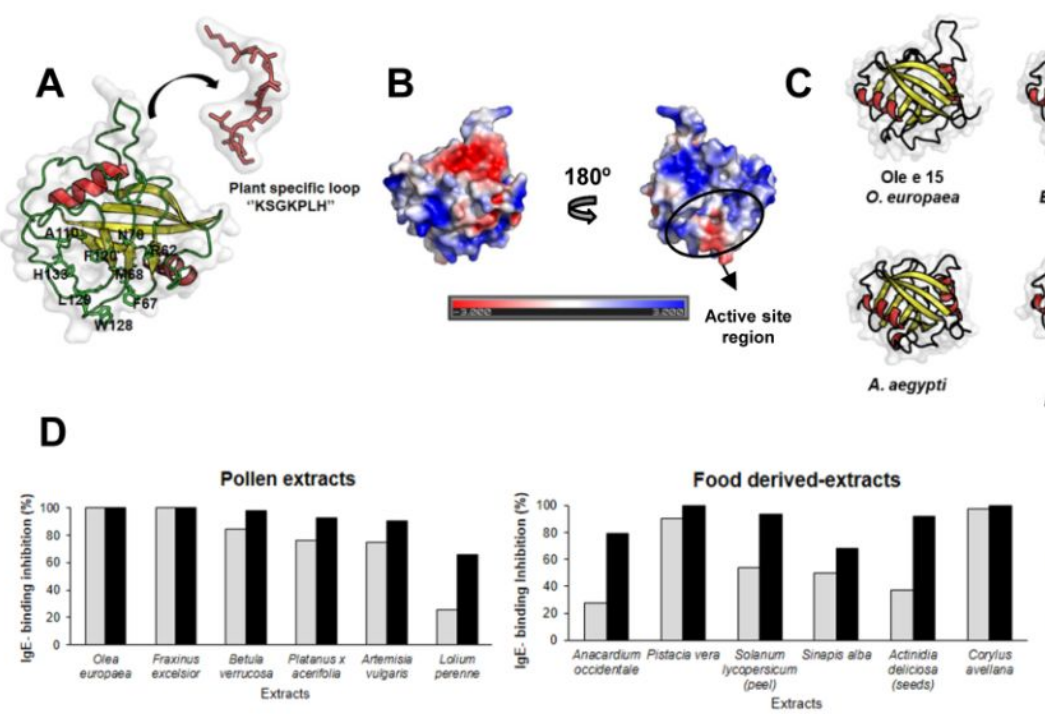

E

Pollen extracts
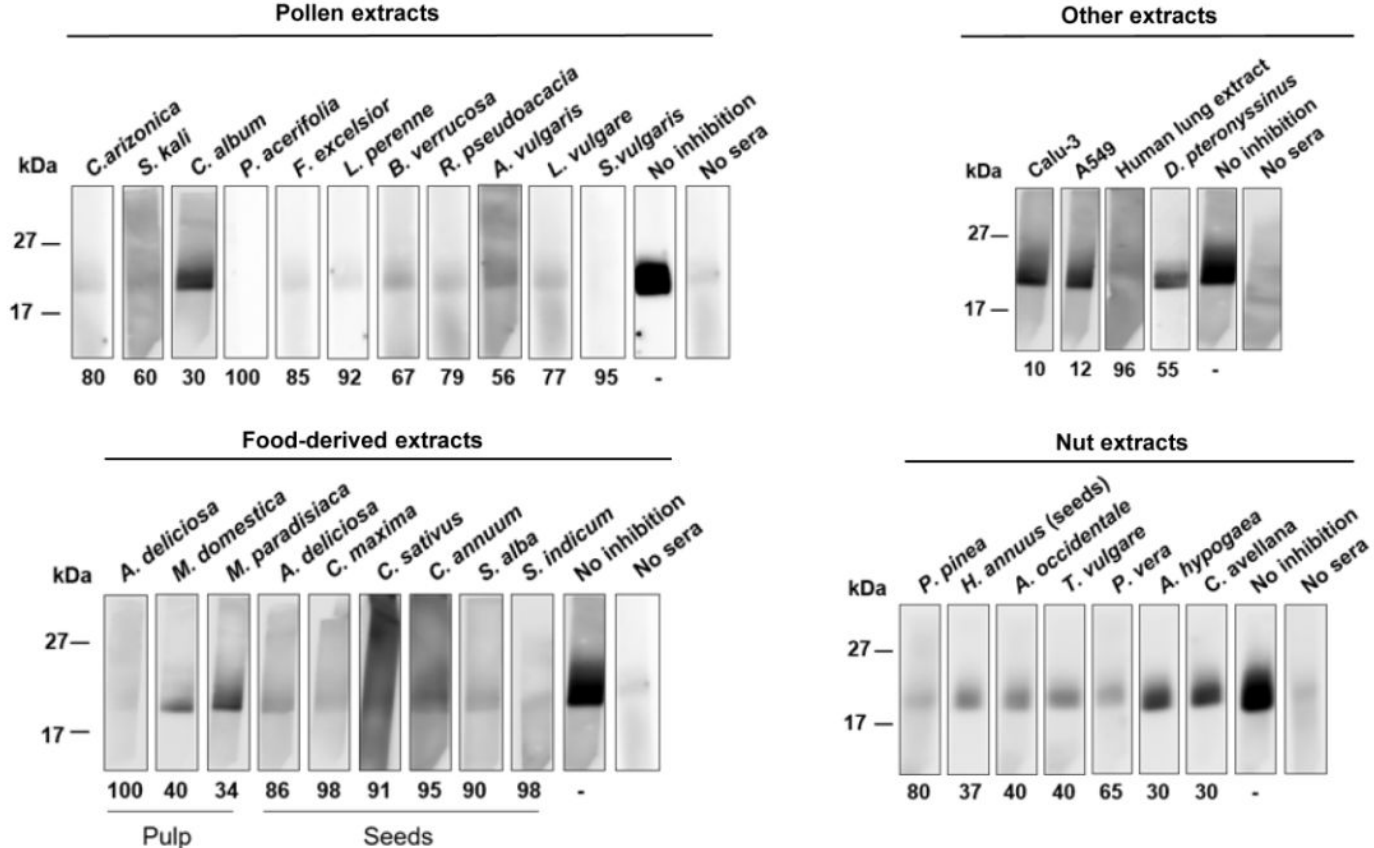

Figure 4. Prediction and comparison of the Ole e 15 structure with other cyclophilins, and analysis of its potential IgE- cross-reactivity. (a) 3D-model of Ole e 15 structure using that of Cat r 1 (PDB: 2mc9) as template. Putative catalytic residues (R62, F67, M68, N70, A110, F120, W128, L129, and H133) and the divergent loop (KSGKLPH) sequence are labeled. (b) predicted electrostatic potential of Ole e 15 using Adaptive Poisson Boltzmann Solver (APBS) program, considering 2 and 78 as dielectric constant values for protein and solvent, respectively. Active site region is highlighted with a circle. Electropositively- and electronegatively-charged regions are colored blue and red, respectively. Neutral regions are colored white. (c) Comparison of the structures of plant (Ole e 15, Bet v 7 and Cat $r$ 1), fungal (Mala s 6) and animal (human PPIA, Der f 29, C. elegans and A. aegypti) cyclophilins. Plant and animal cyclophilins were predicted using structures of Cat $r 1$ (PDB: 2cm9) and human PPIA (PDB: 1OCA) as ExPASy Swiss-Model tool templates, respectively. All structures were generated by PyMOL. (d) Analysis of the IgE inhibition to rOle e 15 was performed by ELISA using an equivolumetric sera pool of olive pollen patients and 25 and $250 \mu \mathrm{g}$ of the indicated extracts as inhibitors. (e) Analysis of the IgE binding to Ole e 15 was performed by WB using an equivolumetric sera pool and $500 \mu \mathrm{g}$ of the indicated protein extracts as inhibitors. All these data suggested a potential implication of olive pollen cyclophilin in cross-reactivity, as it has been shown for other plant and fungal cyclophilins $(40,42,43)$. 


\section{The identification of natural Ole e 15 is hampered by Ole e 1, the main allergen in the olive} pollen extract

Finally, we assessed the behavior of natural Ole e 15 (nOle e 15) in the olive pollen protein extract to determine why this relevant allergen implicated in pollen-pollen, pollen-plant food, or pollen-dust mite cross-reactivity has remained elusive in previous investigations. The most abundant protein in olive pollen extract is the major allergen Ole e 1, which after SDS-PAGE, appears as two main bands of 17 to $18.5 \mathrm{kDa}$ for the non-glycosylated and N-glycosylated isoforms, respectively, with a pI interval (5.0 to 9) for the different isoforms of this allergen. On the other hand, a theoretical mass of $18.1 \mathrm{kDa}$ and $8.7 \mathrm{pI}$ were predicted for natural Ole e 15 , which led us to hypothesize that the presence of Ole e 1 could have masked the identification of Ole e 15 in the extract.

To address this question, WB analysis after 1D and 2D SDS-PAGE of the olive pollen extract with anti-Ole e 1 and anti-Ole e $15 \mathrm{pAb}$ and $\operatorname{IgE}$ inhibition assays confirmed that nOle e 
15 is present as a monomeric protein with an experimental $\mathrm{pI} \sim 8.4$ and apparent molecular mass very similar to glycosylated Ole e 1 (Figure 5a-b). As a consequence, reactivity to nOle e 15 in a cosensitized patient with reactivity to Ole e 1 would remain hidden because of the high protein content of Ole e 1 and its prevalence, and might only be detected if the serum is previously inhibited with Ole e 1 (Figure 5c).

A

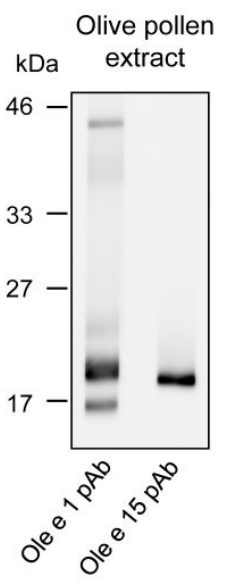

B

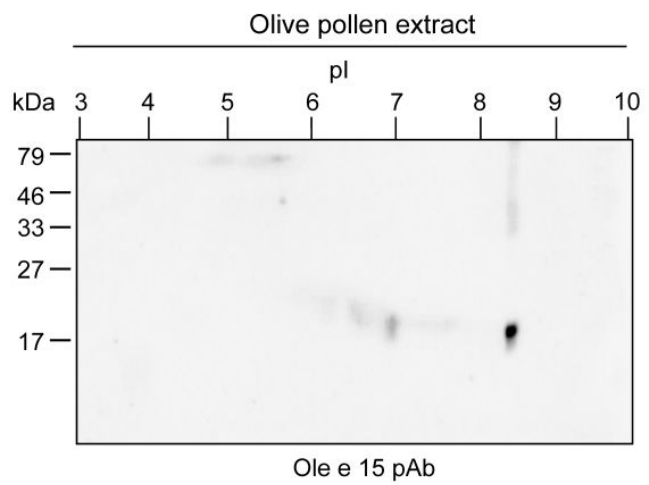

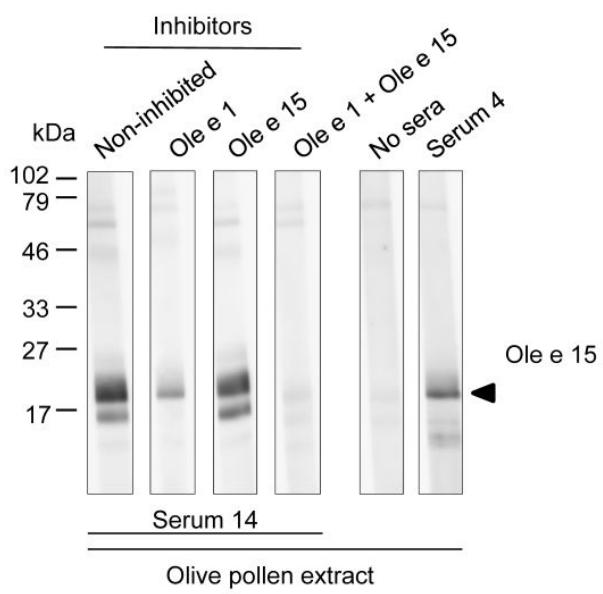

Olive pollen extract

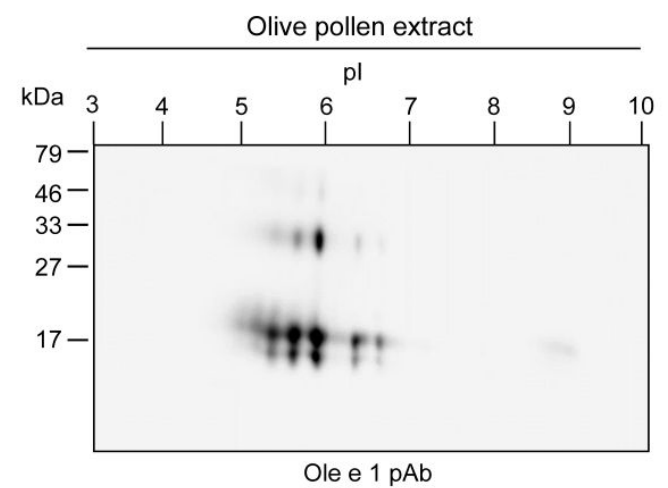

Figure 5. Analysis of Ole e 15 in the olive pollen extract. $(a, b)$ WB analysis after SDS-PAGE of olive pollen extract $(40 \mu \mathrm{g})$ using anti-Ole e 1 and anti-Ole e 15 pAbs. (a) 1D SDS-PAGE. (B) 2D SDS-PAGE. (c) IgE-reactivity analysis after SDS-PAGE and transference to membranes of olive pollen extract. Strips were incubated with the serum \#14, sensitized against Ole e 1 and Ole e 15, after either no inhibition or its IgE inhibition with alternatively $10 \mu \mathrm{g}$ of purified nOle e 1, rOle e 15 or both. No signal was detected without serum incubation (No sera). IgEreactivity of a serum sensitized to Ole e 15 but not to Ole e 1 was also analyzed (Serum \#4). 


\section{Discussion}

Olive pollen is an important allergenic source in those regions with extensive cultivars for olive oil production. The increasing cultivation of this tree in China, India, Australia, Russia, California (US) and multiple countries of South America has led to a growing number of olive pollen allergic patients. Therefore, it will become a top allergenic health problem worldwide in the next years; and thus, the definition of its allergenome will significantly improve the clinical management of olive pollen allergic patients.

Here, to delineate the cultivated olive pollen proteome and its allergenome, we took advantage of the recently reported wild olive genome in combination with a high performance bottom-up LC-MS/MS approach to optimize protein identification. The number of sequences obtained represents the $3.8 \%$ of the complete genome-predicted proteome and is in agreement with published data regarding transcriptomic analysis in olive pollen, in which 1,976 transcripts encoding complete proteins, including isoforms, were found (44). Then, we performed functional annotation of the identified proteins, using a newer version of Blast2GO software and a more up-to-date Gene ontology (GOA) database than the previously used for the genome (14). Our annotation results are the expected for pollen protein functions in germination and tube growth, extremely regulated multistep processes in which secreted or membrane proteins, and enzymes, play a major role (45). In fact, it is also in concordance with other pollen proteomic studies in which most of the proteins were annotated to metabolic processes (46-49). Collectively, we have performed a comprehensive in-depth proteomic study of the olive tree allergenic pollen, and elucidated the distribution of its proteins according to their associated biological function and location, which can be helpful for further studies related to olive pollen biology. Nevertheless, it must be considered that the obtained data is highly dependent on technical and biological issues. For instance, it is known that protein extraction methods in plant proteomics can be determinant to detect hydrophobic membrane proteins and low abundant proteins $(50,51)$. In addition, it has been reported that the use of different solid-phase extraction methods (i.e. ZipTip $v s$ SOLA $\mu$ ) after in-gel trypsin digestion and prior to $\mathrm{nLC}-\mathrm{MS} / \mathrm{MS}$ analysis can increase the coverage in the proteome (52). Moreover, previous studies have demonstrated 
that pollen grains from different olive cultivars differ in the quantity and number of proteoforms, including those of important allergens like Ole e 1, Ole e 2 and Ole e 5 (53-55). This, together with environmental factors like geographic variability in the reached pollen counts, the coexistence of other allergen sources and pollution, as well as human factors like diet, alters the sensitization patterns to olive pollen, especially regarding minor allergens (56). Therefore, further proteomic studies analyzing pollen grains from common cultivars using alternative protein extraction methods would add relevant biological and clinical information to the data here presented.

We focused our study on the identification of allergenic proteins in olive pollen to complete its allergenome. From the 76 allergen families present in the genome-predicted proteome, 47 allergen families were identified in pollen, with 20 non-previously described in this source. Importantly, all of the previously reported allergen families were found $(17-19,57)$. Only the allergen Ole e 6 was not observed. Ole e 6 is present among the potential allergens identified from the genome-predicted proteome (Table S6), and its absence in our proteomic data could be due to either its extremely low abundance in pollen (46), or because of the stringent filters used.

An important feature for some allergenic sources is the high degree of heterogeneity in the same allergen family. According to the WHO/IUIS guidelines for allergen nomenclature, allergenic proteins from the same species are considered isoallergens when they have similar molecular size and a sequence identity of at least $67 \%$; or allergen variants (isoforms) when the proteins display a sequence identity $>90 \%$. It has been shown that isoallergens and allergen variants found in pollen can display different $\operatorname{IgE}$ reactivity $(19,38,53-55,59)$. Moreover, in some sources, proteins of the same allergen family, but not considered isoallergens, can be allergenic (60). In our study, we found more than one protein in 34 of the 47 identified allergen families. In this sense, the Ole e 1-like allergen family was the most heterogeneous (Table 1 and Table S7). Of the 17 proteins found in this family, eight were Ole e 1 variants and, five were Ole e 1 isoallergens. This high degree of Ole e 1 polymorphism had already been described and can vary among different olive cultivars $(55,61)$. The further study of these allergen polymorphisms might help to identify hypoallergenic molecules, which can be used for the 
development of safer immunotherapy strategies. On the other hand, we observed interesting potential allergens which remain to be studied. For example, the presence of a thaumatin-like protein (TLP) similar to Act d 2 was found (Table 1 and Table S7). A member of the TLP family had been described as an olive fruit allergen (Ole e 13) (62), but the presence of other TLPs in olive pollen was unknown. Thus, this finding suggests this protein family could also be allergenic in olive pollen allergic patients, and may be implicated in cross-reactivity with fruits. Additionally, we observed the presence of several allergen families commonly found in the pollen of weeds, grasses and trees (63), such as pectate lyase, papain-like cysteine protease, or cyclophilin. These data validated our workflow to identify potential allergenic proteins, defining the most complete allergenic proteomic profile of olive pollen up to date.

The next goal of this work consisted of the validation of the allergenic potential of some of the newly identified candidate allergens. We selected those protein families reported to be allergenic in at least two allergenic sources, including one non-pollinic, to identify those olive pollen allergens implicated in cross-reactivity. MDH (cytoplasmic and mitochondrial), enolase, and cyclophilin were selected for validation. After their cloning, expression and purification, only cyclophilin was able to bind IgEs from the allergic patients tested. Cyclophilin was named Ole e 15 according to the WHO/IUIS. Remarkably, cytoplasmic and mitochondrial MDH had been previously described as potential allergenic proteins in watermelon, soybean seeds, ash pollen, or yeast; enolase in latex, ragweed pollen, or molds; and cyclophilin in carrot, tomato, birch pollen, molds, and dust mites $(9,39,43,64-72)$. Then, it cannot be discarded that these olive pollen proteins could be reported as allergenic if tested against other allergic populations or if they were expressed in heterologous systems different from E. coli, which would provide post-translational modifications and complex folding.

The last objective of our study was the characterization of olive pollen cyclophilin. Recombinant protein structure exhibited a folding with similar secondary structure as Cat $r$ by far-UV CD (40), indicating that the protein was correctly folded. Regarding its immunological behavior, we observed that the prevalence of Ole e 15 -sensitized patients was about $13 \%$ among olive pollen allergic patients from Córdoba and Madrid, with a majority of them showing high 
IgE levels against the allergen. Although Ole e 15 sensitization was more frequent in pediatric patients $(17.4 \%)$ than in adults $(7.8 \%)$ in both populations, the correlation between Ole e 15 sensitization and pediatric patients was not statistically significant. However, this trend will be assessed in further studies with higher cohorts. After inhibition experiments, we found that Ole e 15 is highly implicated in cross-reactivity with pollen, plant derived food and dust mites. Human cyclophilin, which shows $70 \%$ sequence identity with Ole e 15 , was also able to inhibit the IgE binding to the recombinant protein. Our results are in agreement with a previous study in which plant cyclophilins were predicted to cross-react with animal cyclophilins, due to a high degree of surface conservation (40). This confirmed the broad cross-reactivity of cyclophilins, and extended the physicochemical and immunological knowledge of this pan-allergen to olive pollen. Moreover, as Ole e 15 shares similar physicochemical properties with Ole e 1, the identification of cyclophilin as an allergen has remained elusive by standard techniques. Therefore, the in-depth olive pollen proteomic analysis allowed the identification of this relevant allergen, and suggests that its presence should be assessed in natural preparations to prevent the overestimation of the number of patients allergic to Ole e 1.

In summary, we have performed the most comprehensive proteomic profiling of olive pollen and defined its allergen families. We have also unmasked Ole e 15, a relevant olive pollen allergen belonging to the cyclophilin family with similar physicochemical features to the main olive pollen allergen. After cloning, expression and characterization of Ole e 15, we found that it is a highly potent cross-reactive allergen with plant and animal cyclophilins.

\section{Supporting information:}

The following supporting information is available free of charge at ACS website http://pubs.acs.org

Table S1. Information of the olive pollen allergic patients used in the study.

Table S2. List of the oligonucleotides used in the study.

Table S3. Proteins identified in olive pollen by LC-MS/MS.

Table S4. Olive pollen proteins annotated with GO terms. 


\section{Acknowledgments}

We thank the excellent technical support of Sara Abián. This work was supported by grants co-founded by Fondo Europeo de Desarrollo Regional -FEDER-: SAF2014-53209-R to M.V. and R.B., and SAF2017-86483-R to M.V. from the Ministerio de Economía y Competitividad, and the Thematic Networks and Co-operative Research Centres: RIRAAF Network RD12/0013/0015 and ARADyAL (RD16/0006/0014) from the Instituto de Salud Carlos III (ISCIII). B.R., A.J., A.N, and C.M. acknowledge PI-01119-2016 from the Consejería de Salud (Junta de Andalucía) and the Alergosur Foundation. R.B. also acknowledges the financial support of the PI17CIII/00045 grant from the AES-ISCIII program. P.SS.A. FPU predoctoral contract is supported by the Spanish Ministerio de Educación, Cultura y Deporte. S.B. was a fellow of the Juan de la Cierva program of the Ministerio de Economía y Competitividad (Spain). C.O-S. was supported by a contract of the Programa Operativo de Empleo Juvenil y la Iniciativa de Empleo Juvenil (YEI) with the participation of the Consejería de Educación, Juventud y Deporte de la Comunidad de Madrid y del Fondo Social Europeo.

\section{Disclosure of potential conflict of interest}

All authors declare no conflicts of interest. 


\section{References}

1. $\quad$ McCormick, S., Pollen. Curr Biol 2013, 23, (22), R988-90.

2. Chaturvedi, P.; Ghatak, A.; Weckwerth, W., Pollen proteomics: from stress physiology to developmental priming. Plant Reprod 2016, 29, (1-2), 119-32.

3. Ghatak, A.; Chaturvedi, P.; Paul, P.; Agrawal, G. K.; Rakwal, R.; Kim, S. T.; Weckwerth, W.; Gupta, R., Proteomics survey of Solanaceae family: Current status and challenges ahead. J Proteomics 2017, 169, 41-57.

4. $\quad$ Fíla, J.; Radau, S.; Matros, A.; Hartmann, A.; Scholz, U.; Feciková, J.; Mock, H.-P.; Čapková, V.; Zahedi, R. P.; Honys, D., Phosphoproteomics profiling of tobacco mature pollen and pollen activated in vitro. Mol Cell Proteomics 2016, 15, (4), 1338-1350.

5. $\quad$ Esch, R. E., Grass pollen allergens. Clin Allergy Immunol 2008, 21, 107-26.

6. Asam, C.; Hofer, H.; Wolf, M.; Aglas, L.; Wallner, M., Tree pollen allergens-an update from a molecular perspective. Allergy 2015, 70, (10), 1201-11.

7. Gadermaier, G.; Hauser, M.; Ferreira, F., Allergens of weed pollen: an overview on recombinant and natural molecules. Methods 2014, 66, (1), 55-66.

8. $\quad$ Mas, S.; Torres, M.; Garrido-Arandia, M.; Salamanca, G.; Castro, L.; Barral, P.; Purohit, A.; Pauli, G.; Rodriguez, R.; Batanero, E.; Barderas, R.; Villalba, M., Ash pollen immunoproteomics: identification, immunologic characterization, and sequencing of 6 new allergens. J Allergy Clin Immunol 2014, 133, (3), 923-6 e3.

9. $\quad$ An, S.; Chen, L.; Long, C.; Liu, X.; Xu, X.; Lu, X.; Rong, M.; Liu, Z.; Lai, R., Dermatophagoides farinae allergens diversity identification by proteomics. Mol Cell Proteomics 2013, 12, (7), 1818-28.

10. Choopong, J.; Reamtong, O.; Sookrung, N.; Seesuay, W.; Indrawattana, N.; Sakolvaree, Y.; Chaicumpa, W.; Tungtrongchitr, A., Proteome, allergenome, and novel allergens of house dust mite, Dermatophagoides farinae. J Proteome Res 2016, 15, (2), 422-30.

11. Liu, X.Y.; Yang, K. Y.; Wang, M.Q.; Kwok, J. S.L.; Zeng, X.; Yang, Z.; Xiao, X.J.; Lau, C. P.Y.; Li, Y.; Huang, Z.m.; Ba, J.g.; Yim, A. K.Y.; Ouyang, C.Y.; Ngai, S.M.; Chan, T.F.; Leung, E. L.H.; Liu, L.; Liu, Z.G.; Tsui, S. K.W., Highquality assembly of Dermatophagoides pteronyssinus genome and transcriptome reveals a wide range of novel allergens. J Allergy Clin Immunol 2018, 141, (6), 22682271.e8.

12. Chan, T.F.; Ji, K.M.; Yim, A. K.Y.; Liu, X.Y.; Zhou, J.W.; Li, R.Q.; Yang, K. Y.; Li, J.; Li, M.; Law, P. T.W.; Wu, Y.L.; Cai, Z.L.; Qin, H.; Bao, Y.; Leung, R. K.K.; Ng, P. K.S.; Zou, J.; Zhong, X.J.; Ran, P.X.; Zhong, N.S.; Liu, Z.G.; Tsui, S. K.W., The draft genome, transcriptome, and microbiome of Dermatophagoides farinae reveal a broad spectrum of dust mite allergens. J Allergy Clin Immunol 2015, 135, (2), 539-548.

13. Nugraha, R.; Kamath, S. D.; Johnston, E.; Zenger, K. R.; Rolland, J. M.; O'Hehir, R. E.; Lopata, A. L., Rapid and comprehensive discovery of unreported shellfish allergens using largescale transcriptomic and proteomic resources. J Allergy Clin Immunol 2018, 141, (4), 15011504.e8.

14. Unver, T.; Wu, Z.; Sterck, L.; Turktas, M.; Lohaus, R.; Li, Z.; Yang, M.; He, L.; Deng, T.; Escalante, F. J.; Llorens, C.; Roig, F. J.; Parmaksiz, I.; Dundar, E.; Xie, F.; Zhang, B.; Ipek, A.; Uranbey, S.; Erayman, M.; Ilhan, E.; Badad, O.; Ghazal, H.; Lightfoot, D. A.; Kasarla, P.; Colantonio, V.; Tombuloglu, H.; Hernandez, P.; Mete, N.; Cetin, O.; Van Montagu, M.; Yang, H.; Gao, Q.; Dorado, G.; Van de Peer, Y., Genome of wild olive and the evolution of oil biosynthesis. Proc Natl Acad Sci U S A 2017, 114, (44), E9413-E9422.

15. Diez, C. M.; Trujillo, I.; Martinez-Urdiroz, N.; Barranco, D.; Rallo, L.; Marfil, P.; Gaut, B. S., Olive domestication and diversification in the Mediterranean Basin. New Phytol 2015, 206, (1), 436-47.

16. D'Amato, G.; Lobefalo, G., Allergenic pollens in the southern Mediterranean area. $J$ Allergy Clin Immunol 1989, 83, (1), 116-22.

17. Villalba, M.; Rodriguez, R.; Batanero, E., The spectrum of olive pollen allergens. From structures to diagnosis and treatment. Methods 2014, 66, (1), 44-54.

18. Oeo-Santos, C.; Mas, S.; Benede, S.; Lopez-Lucendo, M.; Quiralte, J.; Blanca, M.; Mayorga, C.; Villalba, M.; Barderas, R., A recombinant isoform of the Ole e 7 olive pollen 
allergen assembled by de novo mass spectrometry retains the allergenic ability of the natural allergen. J Proteomics 2018, 187, 39-46.

19. Oeo-Santos, C.; Mas, S.; Quiralte, J.; Colas, C.; Blanca, M.; Fernandez, J.; Feo-Brito, F.; Villalba, M.; Barderas, R., A hypoallergenic polygalacturonase isoform from olive pollen is implicated in pollen-pollen cross-reactivity. Int Arch Allergy Immunol 2018, 177, (4), 290-301. 20. Boldogh, I.; Bacsi, A.; Choudhury, B. K.; Dharajiya, N.; Alam, R.; Hazra, T. K.; Mitra, S.; Goldblum, R. M.; Sur, S., ROS generated by pollen NADPH oxidase provide a signal that augments antigen-induced allergic airway inflammation. $J$ Clin Invest 2005, 115, (8), 2169-79. 21. Kauffman, H. F.; Tamm, M.; Timmerman, J. A.; Borger, P., House dust mite major allergens Der $\mathrm{p} 1$ and Der $\mathrm{p} 5$ activate human airway-derived epithelial cells by proteasedependent and protease-independent mechanisms. Clin Mol Allergy 2006, 4, 5.

22. Matsumura, Y., Role of allergen source-derived proteases in sensitization via airway epithelial cells. $J$ Allergy (Cairo) 2012, 2012, 903659.

23. Mari, A.; Rasi, C.; Palazzo, P.; Scala, E., Allergen databases: Current status and perspectives. Currr Allergy Asthma Rep 2009, 9, (5), 376-383.

24. Radauer, C.; Bublin, M.; Wagner, S.; Mari, A.; Breiteneder, H., Allergens are distributed into few protein families and possess a restricted number of biochemical functions. $J$ Allergy Clin Immunol 2008, 121, (4), 847-52 e7.

25. Radauer, C.; Nandy, A.; Ferreira, F.; Goodman, R. E.; Larsen, J. N.; Lidholm, J.; Pomes, A.; Raulf-Heimsoth, M.; Rozynek, P.; Thomas, W. R.; Breiteneder, H., Update of the WHO/IUIS Allergen Nomenclature Database based on analysis of allergen sequences. Allergy 2014, 69, (4), 413-9.

26. Barral, P.; Suarez, C.; Batanero, E.; Alfonso, C.; Alche Jde, D.; Rodriguez-Garcia, M. I.; Villalba, M.; Rivas, G.; Rodriguez, R., An olive pollen protein with allergenic activity, Ole e 10 , defines a novel family of carbohydrate-binding modules and is potentially implicated in pollen germination. Biochem J 2005, 390, (Pt 1), 77-84.

27. Pelaez-Garcia, A.; Barderas, R.; Batlle, R.; Vinas-Castells, R.; Bartolome, R. A.; Torres, S.; Mendes, M.; Lopez-Lucendo, M.; Mazzolini, R.; Bonilla, F.; Garcia de Herreros, A.; Casal, J. I., A proteomic analysis reveals that Snail regulates the expression of the nuclear orphan receptor Nuclear Receptor Subfamily 2 Group F Member 6 (Nr2f6) and interleukin 17 (IL-17) to inhibit adipocyte differentiation. Mol Cell Proteomics 2015, 14, (2), 303-15.

28. Pelaez-Garcia, A.; Barderas, R.; Mendes, M.; Lopez-Lucendo, M.; Sanchez, J. C.; Garcia de Herreros, A.; Casal, J. I., Data from proteomic characterization of the role of Snail1 in murine mesenchymal stem cells and 3T3-L1 fibroblasts differentiation. Data Brief 2015, 4, 60613.

29. Kall, L.; Canterbury, J. D.; Weston, J.; Noble, W. S.; MacCoss, M. J., Semi-supervised learning for peptide identification from shotgun proteomics datasets. Nat Methods 2007, 4, (11), 923-5.

30. Gotz, S.; Garcia-Gomez, J. M.; Terol, J.; Williams, T. D.; Nagaraj, S. H.; Nueda, M. J.; Robles, M.; Talon, M.; Dopazo, J.; Conesa, A., High-throughput functional annotation and data mining with the Blast2GO suite. Nucleic Acids Res 2008, 36, (10), 3420-35.

31. Madeira, F.; Park, Y. M.; Lee, J.; Buso, N.; Gur, T.; Madhusoodanan, N.; Basutkar, P.; Tivey, A. R. N.; Potter, S. C.; Finn, R. D.; Lopez, R., The EMBL-EBI search and sequence analysis tools APIs in 2019. Nucleic Acids Res 2019.

32. Zerbino, D. R.; Achuthan, P.; Akanni, W.; Amode, M. R.; Barrell, D.; Bhai, J.; Billis, K.; Cummins, C.; Gall, A.; Giron, C. G.; Gil, L.; Gordon, L.; Haggerty, L.; Haskell, E.; Hourlier, T.; Izuogu, O. G.; Janacek, S. H.; Juettemann, T.; To, J. K.; Laird, M. R.; Lavidas, I.; Liu, Z.; Loveland, J. E.; Maurel, T.; McLaren, W.; Moore, B.; Mudge, J.; Murphy, D. N.; Newman, V.; Nuhn, M.; Ogeh, D.; Ong, C. K.; Parker, A.; Patricio, M.; Riat, H. S.; Schuilenburg, H.; Sheppard, D.; Sparrow, H.; Taylor, K.; Thormann, A.; Vullo, A.; Walts, B.; Zadissa, A.; Frankish, A.; Hunt, S. E.; Kostadima, M.; Langridge, N.; Martin, F. J.; Muffato, M.; Perry, E.; Ruffier, M.; Staines, D. M.; Trevanion, S. J.; Aken, B. L.; Cunningham, F.; Yates, A.; Flicek, P., Ensembl 2018. Nucleic Acids Res 2018, 46, (D1), D754-d761. 
33. Durinck, S.; Spellman, P. T.; Birney, E.; Huber, W., Mapping identifiers for the integration of genomic datasets with the R/Bioconductor package biomaRt. Nat Protoc 2009, 4, (8), 1184-91.

34. Jones, P.; Binns, D.; Chang, H. Y.; Fraser, M.; Li, W.; McAnulla, C.; McWilliam, H.; Maslen, J.; Mitchell, A.; Nuka, G.; Pesseat, S.; Quinn, A. F.; Sangrador-Vegas, A.; Scheremetjew, M.; Yong, S. Y.; Lopez, R.; Hunter, S., InterProScan 5: genome-scale protein function classification. Bioinformatics 2014, 30, (9), 1236-40.

35. Kanehisa, M.; Goto, S., KEGG: kyoto encyclopedia of genes and genomes. Nucleic Acids Res 2000, 28, (1), 27-30.

36. Petersen, T. N.; Brunak, S.; von Heijne, G.; Nielsen, H., SignalP 4.0: discriminating signal peptides from transmembrane regions. Nat Methods 2011, 8, (10), 785-6.

37. Baker, N. A.; Sept, D.; Joseph, S.; Holst, M. J.; McCammon, J. A., Electrostatics of nanosystems: application to microtubules and the ribosome. Proc Natl Acad Sci US A 2001, 98, (18), 10037-41.

38. Mas, S.; Oeo-Santos, C.; Cuesta-Herranz, J.; Diaz-Perales, A.; Colas, C.; Fernandez, J.; Barber, D.; Rodriguez, R.; de Los Rios, V.; Barderas, R.; Villalba, M., A relevant IgE-reactive $28 \mathrm{kDa}$ protein identified from Salsola kali pollen extract by proteomics is a natural degradation product of an integral 47kDa polygalaturonase. Biochim Biophys Acta 2017, 1865, (8), 1067 1076.

39. Poncet, P.; Senechal, H.; Clement, G.; Purohit, A.; Sutra, J. P.; Desvaux, F. X.; Wal, J. M.; Pauli, G.; Peltre, G.; Gougeon, M. L., Evaluation of ash pollen sensitization pattern using proteomic approach with individual sera from allergic patients. Allergy 2010, 65, (5), 571-80. 40. Ghosh, D.; Mueller, G. A.; Schramm, G.; Edwards, L. L.; Petersen, A.; London, R. E.; Haas, H.; Gupta Bhattacharya, S., Primary identification, biochemical characterization, and immunologic properties of the allergenic pollen cyclophilin cat R 1. J Biol Chem 2014, 289, (31), 21374-85.

41. Davis, T. L.; Walker, J. R.; Campagna-Slater, V.; Finerty, P. J., Jr.; Paramanathan, R.; Bernstein, G.; MacKenzie, F.; Tempel, W.; Ouyang, H.; Lee, W. H.; Eisenmesser, E. Z.; DhePaganon, S., Structural and Biochemical Characterization of the Human Cyclophilin Family of Peptidyl-Prolyl Isomerases. PLOS Biol 2010, 8, (7), e1000439.

42. Glaser, A. G.; Limacher, A.; Fluckiger, S.; Scheynius, A.; Scapozza, L.; Crameri, R., Analysis of the cross-reactivity and of the $1.5 \mathrm{~A}$ crystal structure of the Malassezia sympodialis Mala s 6 allergen, a member of the cyclophilin pan-allergen family. Biochem J 2006, 396, (1), 41-9.

43. Cadot, P.; Nelles, L.; Srahna, M.; Dilissen, E.; Ceuppens, J. L., Cloning and expression of the cyclophilin Bet $\mathrm{v}$ 7, and analysis of immunological cross-reactivity among the cyclophilin A family. Mol Immunol 2006, 43, (3), 226-35.

44. Carmona, R.; Zafra, A.; Seoane, P.; Castro, A. J.; Guerrero-Fernández, D.; CastilloCastillo, T.; Medina-García, A.; Cánovas, F. M.; Aldana-Montes, J. F.; Navas-Delgado, I.; Alché, J. d. D.; Claros, M. G., ReprOlive: a database with linked data for the olive tree (Olea europaea L.) reproductive transcriptome. Front Plant Sci 2015, 6, (625).

45. Dresselhaus, T.; Franklin-Tong, N., Male-Female crosstalk during pollen germination, tube growth and guidance, and double fertilization. Mol Plant 2013, 6, (4), 1018-1036.

46. Kawakami, T.; Darby, B. J.; Ungerer, M. C., Transcriptome resources for the perennial sunflower Helianthus maximiliani obtained from ecologically divergent populations. Mol Ecol Resour 2014, 14, (4), 812-9.

47. Sheoran, I. S.; Sproule, K. A.; Olson, D. J.; Ross, A. R.; Sawhney, V. K., Proteome profile and functional classification of proteins in Arabidopsis thaliana (Landsberg erecta) mature pollen. Sexual Plant Reproduction 2006, 19, (4), 185-196.

48. Zhang, J.; Wu, L. S.; Fan, W.; Zhang, X. L.; Jia, H. X.; Li, Y.; Yin, Y. F.; Hu, J. J.; Lu, M. Z., Proteomic analysis and candidate allergenic proteins in Populus deltoides CL. "2KEN8" mature pollen. Front Plant Sci 2015, 6, 548.

49. Pablos, I.; Eichhorn, S.; Briza, P.; Asam, C.; Gartner, U.; Wolf, M.; Ebner, C.; Bohle, B.; Arora, N.; Vieths, S.; Ferreira, F.; Gadermaier, G., Proteomic profiling of the weed feverfew, a neglected pollen allergen source. Sci Rep 2017, 7, (1), 6049. 
50. Niu, L.; Yuan, H.; Gong, F.; Wu, X.; Wang, W., Protein extraction methods shape much of the extracted proteomes. Front Plant Sci 2018, 9, (802).

51. Karthikaichamy, A.; Deore, P.; Rai, V.; Bulach, D.; Beardall, J.; Noronha, S.; Srivastava, S., Time for multiple extraction methods in proteomics? A comparison of three protein extraction methods in the Eustigmatophyte Alga Microchloropsis gaditana CCMP526. Omics 2017, 21, (11), 678-683.

52. Schmelter, C.; Funke, S.; Treml, J.; Beschnitt, A.; Perumal, N.; Manicam, C.; Pfeiffer, N.; Grus, F. H., Comparison of two solid-phase extraction (SPE) methods for the identification and quantification of porcine retinal protein markers by LC-MS/MS. Int J Mol Sci 2018, 19, (12).

53. Jimenez-Lopez, J. C.; Morales, S.; Castro, A. J.; Volkmann, D.; Rodriguez-Garcia, M. I.; Alche, J. D., Characterization of profilin polymorphism in pollen with a focus on multifunctionality. PLoS One 2012, 7, (2), e30878.

54. Alche, J. D.; Castro, A. J.; Jimenez-Lopez, J. C.; Morales, S.; Zafra, A.; HammanKhalifa, A. M.; Rodriguez-Garcia, M. I., Differential characteristics of olive pollen from different cultivars: biological and clinical implications. J Investig Allergol Clin Immunol 2007, 17 Suppl 1, 17-23.

55. Hamman-Khalifa, A.; Castro, A. J.; Jimenez-Lopez, J. C.; Rodriguez-Garcia, M. I.; Alche Jde, D., Olive cultivar origin is a major cause of polymorphism for Ole e 1 pollen allergen. BMC Plant Biol 2008, 8, 10.

56. Barber, D.; Moreno, C.; Ledesma, A.; Serrano, P.; Galan, A.; Villalba, M.; Guerra, F.; Lombardero, M.; Rodriguez, R., Degree of olive pollen exposure and sensitization patterns. Clinical implications. J Investig Allergol Clin Immunol 2007, 17 Suppl 1, 11-6.

57. Rejon, J. D.; Delalande, F.; Schaeffer-Reiss, C.; Alche, J. D.; Rodriguez-Garcia, M. I.; Van Dorsselaer, A.; Castro, A. J., The pollen coat proteome: at the cutting edge of plant reproduction. Proteomes 2016, 4, (1).

58. Barral, P.; Tejera, M. L.; Trevino, M. A.; Batanero, E.; Villalba, M.; Bruix, M.; Rodriguez, R., Recombinant expression of Ole e 6, a Cys-enriched pollen allergen, in Pichia pastoris yeast: detection of partial oxidation of methionine by NMR. Protein Expr Purif 2004, 37, (2), 336-43.

59. Seutter von Loetzen, C.; Reuter, A.; Spiric, J.; Schulenborg, T.; Bellinghausen, I.; Volker, E.; Vogel, L.; Rosch, P.; Schiller, D., Quality and potency profile of eight recombinant isoallergens, largely mimicking total Bet v 1-specific IgE binding of birch pollen. Clin Exp Allergy 2019, 49, (5), 712-723.

60. Dubiela, P.; Kabasser, S.; Smargiasso, N.; Geiselhart, S.; Bublin, M.; Hafner, C.; Mazzucchelli, G.; Hoffmann-Sommergruber, K., Jug r 6 is the allergenic vicilin present in walnut responsible for IgE cross-reactivities to other tree nuts and seeds. Sci Rep 2018, 8, (1), 11366.

61. Villalba, M.; Batanero, E.; Monsalve, R. I.; Gonzalez de la Pena, M. A.; Lahoz, C.; Rodriguez, R., Cloning and expression of Ole e I, the major allergen from olive tree pollen. Polymorphism analysis and tissue specificity. J Biol Chem 1994, 269, (21), 15217-22. 62. Torres, M.; Alvarez-Garcia, E.; Bartra, J.; Alcantara, M.; Palomares, O.; Villalba, M.; Rodriguez, R., The allergenic structure of the thaumatin-like protein Ole e 13 is degraded by processing of raw olive fruits. J Investig Allergol Clin Immunol 2014, 24, (3), 162-8. 63. Pablos, I.; Wildner, S.; Asam, C.; Wallner, M.; Gadermaier, G., Pollen Allergens for Molecular Diagnosis. Curr Allergy Asthma Rep 2016, 16, (4), 31.

64. Pastor, C.; Cuesta-Herranz, J.; Cases, B.; Perez-Gordo, M.; Figueredo, E.; de las Heras, M.; Vivanco, F., Identification of major allergens in watermelon. Int Arch Allergy Immunol 2009, 149, (4), 291-8.

65. Kim, J.-H.; Ahn, K.-M.; Kim, W.; Han, Y.; Kim, Y.-R.; Lee, S.; Kim, H.-Y., Identification of novel IgE-binding soybean allergens using serological analysis of a recombinant cDNA expression library (SEREX). Food Sci Biotechnol 2014, 23, (4), 1037-1042. 66. Yoshimi, O.; Masanobu, K.; Hiroshi, Y.; Akemi, S.; Eiko, S.-K.; Susumu, T.; Takashi, H.-O.; Tomoko, Y.; Katsuhisa, U.; Hideyo, Y.; Kazuo, A.; Ikunoshin, K.; Kazutoh, T., Twodimensional electrophoresis of Malassezia allergens for atopic dermatitis and isolation of Mal 
f 4 homologs with mitochondrial malate dehydrogenase. Eur J Biochem 1999, 261, (1), 148154.

67. Wagner, S.; Breiteneder, H.; Simon-Nobbe, B.; Susani, M.; Krebitz, M.; Niggemann, B.; Brehler, R.; Scheiner, O.; Hoffmann-Sommergruber, K., Hev b 9, an enolase and a new cross-reactive allergen from hevea latex and molds. Purification, characterization, cloning and expression. Eur J Biochem 2000, 267, (24), 7006-14.

68. Zhao, F.; Elkelish, A.; Durner, J.; Lindermayr, C.; Winkler, J. B.; Rusmall io, R. F.; Behrendt, H.; Traidl-Hoffmann, C.; Holzinger, A.; Kofler, W.; Braun, P.; von Toerne, C.;

Hauck, S. M.; Ernst, D.; Frank, U., Common ragweed (Ambrosia artemisiifolia L.): allergenicity and molecular characterization of pollen after plant exposure to elevated $\mathrm{NO}_{2}$. Plant Cell Environ 2016, 39, (1), 147-64.

69. Kespohl, S.; Raulf, M., Mould allergens: Where do we stand with molecular allergy diagnostics?: Part 13 of the series Molecular Allergology. Allergo J Int 2014, 23, (4), 120-125.

70. Fujita, C.; Moriyama, T.; Ogawa, T., Identification of cyclophilin as an IgE-binding protein from carrots. Int Arch Allergy Immunol 2001, 125, (1), 44-50.

71. Fluckiger, S.; Fijten, H.; Whitley, P.; Blaser, K.; Crameri, R., Cyclophilins, a new family of cross-reactive allergens. Eur J Immunol 2002, 32, (1), 10-7.

72. Welter, S.; Dolle, S.; Lehmann, K.; Schwarz, D.; Weckwerth, W.; Worm, M.; Franken, P., Pepino mosaic virus infection of tomato affects allergen expression, but not the allergenic potential of fruits. PLoS One 2013, 8, (6), e65116. 


\section{For TOC Only}

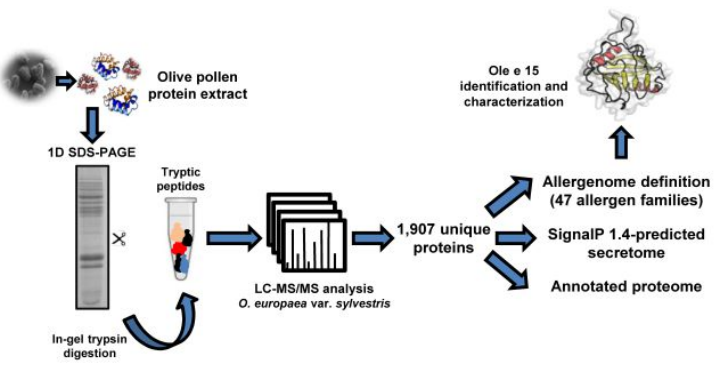

